\title{
Globules and pillars in Cygnus $X$
}

\section{Herschel ${ }^{\star}$ far-infrared imaging of the Cygnus OB2 environment}

\author{
N. Schneider ${ }^{1,2,3}$, S. Bontemps ${ }^{1,2}$, F. Motte ${ }^{4,5}$, A. Blazere ${ }^{1,2}$, Ph. André ${ }^{4}$, L. D. Anderson ${ }^{6}$, D. Arzoumanian ${ }^{4,7}$, \\ F. Comerón ${ }^{8}$, P. Didelon ${ }^{4}$, J. Di Francesco ${ }^{9,10}$, A. Duarte-Cabral ${ }^{11}$, M. G. Guarcello ${ }^{12,13}$, M. Hennemann ${ }^{4}$, T. Hill ${ }^{14}$, \\ V. Könyves ${ }^{4}$, A. Marston ${ }^{15}$, V. Minier ${ }^{4}$, K. L. J. Rygl ${ }^{16}$, M. Röllig ${ }^{3}$, A. Roy ${ }^{4}$, L. Spinoglio ${ }^{17}$, P. Tremblin ${ }^{18}$, \\ G. J. White ${ }^{19,20}$, and N. J. Wright ${ }^{21}$
}

1 Université Bordeaux, LAB, UMR 5804, 33270 Floirac, France

2 CNRS, LAB, UMR 5804, 33270 Floirac, France

3 I. Physik. Institut, University of Cologne, Zülpicher Str. 77, 50937 Koeln, Germany e-mail: nschneid@ph1. uni-koeln.de

${ }^{4}$ IRFU/SAp CEA/DSM, Laboratoire AIM CNRS - Université Paris Diderot, 91191 Gif-sur-Yvette, France

5 IPAG, University Grenoble Alpes, 38000 Grenoble, France

6 Dep. of Physics, West Virginia University, WV 26506, USA

7 IAS, CNRS/Université Paris-Sud 11, 91405 Orsay, France

8 ESO, Alonso de Cordova 3107, Vitacura, Santiago 19, Chile

9 Dep. of Physics and Astronomy, University of Victoria, Victoria, BC V8W 2Y2, Canada

10 NRCC, Victoria, BC V9E 2E7, Canada

11 Astrophysics Group, University of Exeter, EX4 4QL Exeter, UK

12 INAF Osservatorio Astronomico di Palermo, 90134 Palermo, Italy

13 Smithsonian Astrophysical Observatory, Cambridge, MA 02138, USA

14 Joint ALMA Observatory, 3107 Alonso de Cordova, Vitacura, Santiago 19, Chile

15 Herschel Science Centre, European Space Astronomy Centre (ESAC)/ESA, Villanueva de la Canada, Spain

16 INAF-ORA, via P.Gobetti 101, 40129 Bologna, Italy

17 INAF-IAPS, via Fosso del Cavaliere 100, 00133 Roma, Italy

18 Maison de la Simulation, CEA-CNRS-INRIA-UPS-UVSQ, USR 3441, CEA Saclay, 91191 Gif-sur-Yvette, France

19 Department of Physics \& Astronomy, The Open University, Milton Keynes MK7 6AA, UK

${ }^{20}$ RALSpace, The Rutherford Appleton Laboratory, Chilton, Didcot, Oxfordshire OX11 0NL, UK

${ }^{21}$ Centre for Astrophysics Research, University of Hertfordshire, Hatfield, AL10 9AB, UK

Received 17 February 2016/ Accepted 5 April 2016

\begin{abstract}
The radiative feedback of massive stars on molecular clouds creates pillars, globules and other features at the interface between the $\mathrm{H}$ II region and molecular cloud. Optical and near-infrared observations from the ground as well as with the Hubble or Spitzer satellites have revealed numerous examples of such cloud structures. We present here Herschel far-infrared observations between $70 \mu \mathrm{m}$ and $500 \mu \mathrm{m}$ of the immediate environment of the rich Cygnus OB2 association, performed within the Herschel imaging survey of OB Young Stellar objects (HOBYS) program. All of the observed irradiated structures were detected based on their appearance at $70 \mu \mathrm{m}$, and have been classified as pillars, globules, evaporating gasous globules (EGGs), proplyd-like objects, and condensations. From the $70 \mu \mathrm{m}$ and $160 \mu \mathrm{m}$ flux maps, we derive the local far-ultraviolet (FUV) field on the photon dominated surfaces. In parallel, we use a census of the O-stars to estimate the overall FUV-field, that is $10^{3}-10^{4} G_{0}$ (Habing field) close to the central OB cluster (within $10 \mathrm{pc}$ ) and decreases down to a few tens $G_{0}$, in a distance of $50 \mathrm{pc}$. From a spectral energy distribution (SED) fit to the four longest Herschel wavelengths, we determine column density and temperature maps and derive masses, volume densities and surface densities for these structures. We find that the morphological classification corresponds to distinct physical properties. Pillars and globules are massive $\left(\sim 500 M_{\odot}\right)$ and large (equivalent radius $\left.r \sim 0.6 \mathrm{pc}\right)$ structures, corresponding to what is defined as "clumps" for molecular clouds. EGGs and proplyd-like objects are smaller $(r \sim 0.1$ and $0.2 \mathrm{pc})$ and less massive $\left(\sim 10\right.$ and $\left.\sim 30 M_{\odot}\right)$. Cloud condensations are small $(\sim 0.1 \mathrm{pc})$, have an average mass of $35 M_{\odot}$, are dense $\left(\sim 6 \times 10^{4} \mathrm{~cm}^{-3}\right)$, and can thus be described as molecular cloud "cores". All pillars and globules are oriented toward the Cyg OB2 association center and have the longest estimated photoevaporation lifetimes, a few million years, while all other features should survive less than a million years. These lifetimes are consistent with that found in simulations of turbulent, UV-illuminated clouds. We propose a tentative evolutionary scheme in which pillars can evolve into globules, which in turn then evolve into EGGs, condensations and proplyd-like objects.
\end{abstract}

Key words. ISM: clouds - ISM: individual objects: Cygnus X

\footnotetext{
^ Herschel is an ESA space observatory with science instruments provided by European-led Principal Investigator consortia and with important participation from NASA.
}

\section{Introduction}

In the environment of high-mass stars, a rich diversity of large and small dusty gas condensations are produced under the 
influence of ionizing radiation. These structures have mainly been detected through optical observations (see, e.g., Herbig 1974; Schneps et al. 1980; White et al. 1997 for an overview). Column-like features were named "elephant-trunks", and more isolated globule-shaped objects were named "teardrop" or "cometary" globules. In particular Hubble and Spitzer revealed manifold examples and the detailed structure of pillars in Galactic $\mathrm{H}_{\text {II }}$ regions, like the famous Pillars of Creation in M16 or the giant dust pillars in Carina. Pillars have a column shape, are attached to their native molecular cloud, and cover scales from $\sim 0.5 \mathrm{pc}$ up to a few pc in length. Globules (on similar size scales) are isolated and have a characteristic head-tail structure. Lowmass star formation can take place in pillars (Hester et al. 1996; White et al. 1999), and globules (e.g., Sugitani et al. 2002), but there are only a few examples of high-mass stars in globules. Intermediate-mass early B-stars have been found in a globule in Cygnus (Schneider et al. 2012a; Djupvik et al., in prep.), which is part of this study.

On smaller size scales $(<0.5 \mathrm{pc})$, there is a whole collection of different expressions for observed features: small globules (evaporating gaseous globules, hereafter EGGs) are interpreted as fragments of a cloud (e.g., Smith et al. 2003) which possibly form stars (McCaughrean \& Andersen 2002), while proplyds (O'Dell et al. 1993) are evaporating circumstellar disks. After the discovery of proplyds in the Orion Nebula (e.g., Laques \& Vidal 1979) they were searched for in the vicinity of other OB associations. Wright et al. (2012) found proplyd-like objects in the immediate environment of the Cyg OB2 association but doubted that these are all disk-features. Another group of tiny $(<0.05 \mathrm{pc})$ isolated condensations were detected by Gahm et al. (2007) in various Galactic $\mathrm{H}_{\text {II }}$ regions who named them globulettes.

With Herschel far-infrared (FIR) imaging of high-mass star-forming regions within the Herschel imaging survey of OB Young Stellar objects (HOBYS) ${ }^{1}$ key program (Motte et al. 2010), it is now possible to detect these features in the FIR (provided that they are not too small) and also to determine systematically their physical properties such as size, mass, and temperature. At the same time, Herschel observations can provide a census of pre- and protostellar sources, and thus link the properties of pillars and globules to star formation within them. So far, Herschel observations have shown that the majority of low-mass stars form within filaments (e.g., André et al. 2010, 2014), and OB clusters where filaments merge (e.g., Schneider et al. 2012b; Hennemann et al. 2012). It is not clear whether star formation in pillars, globules, proplyds etc. follows the same path. It is possible that dense filaments and accretion flows are shaped by UV radiation into the form of pillars, as was recently shown in numerical simulations with stellar feedback processes (Dale et al. 2014). These pillar-like features can then fragment into smaller units that evolve under the influence of radiation into globules, EGGs, condensations, proplyds, etc., depending on the intial preexisting density structure, and finally form stars. The more classical view is that large-scale compression of an expanding $\mathrm{H}$ II region on a molecular cloud surface creates pillars that also evolve into globules, EGGs, condensations etc. Various studies (e.g., Lefloch \& Lazareff 1994; Williams et al. 2001; Miao et al. 2006, 2009) have shown that instabilities in the $\mathrm{H}_{\text {II }}$ region/molecular cloud interface create bright-rimmed clouds and pillars that can detach to form isolated globules. The importance of the turbulent gas structure was recognized by Gritschneder et al. (2009, 2010). Tremblin et al. (2012a,b) have shown that the turbulent density structure of molecular clouds can lead to local curvatures of the

PIs: Motte, Zavagno, Bontemps; www . herschel.fr/cea/hobys dense shell formed by the ionization compression, which may develop into pillars that can subsequently detach from the cloud. When the turbulent ram pressure of the molecular gas is larger than the ionized-gas pressure, globules can form.

In any case, both star-formation schemes outlined above produce isolated stars and the difference between them lies mainly in its primoridal phase. It is thus very difficult, perhaps impossible, to find observational which would allow us to discriminate between the two scenarios described.

Several HOBYS studies have already observed the H II region/molecular cloud interface, including pillars, globules, and $\mathrm{H}_{\mathrm{II}}$ bubbles, and how these are impacted by OB-clusters through heating (e.g., Schneider et al. 2010; Hill et al. 2012; Anderson et al. 2012; Didelon et al. 2015), external compression (Zavagno et al. 2010; Hill et al. 2011; Minier et al. 2013; Tremblin et al. 2014), and ionization (Deharveng et al. 2012; Tremblin et al. 2013). In this paper, we focus on investigating the immediate environment of the Cyg OB2 association in the so-called Cygnus X region (see, e.g., Reipurth \& Schneider 2008 for an overview). Cygnus $\mathrm{X}$ is a large $\sim 10$ degree wide radio emission feature (Piddington \& Minnett 1952) composed of numerous individual $\mathrm{H}_{\text {II }}$ regions. A major problem is the uncertainty in distances because we look down a spiral arm, with the resulting confusion of regions as near as a few hundred parsec with others at 1-2 kpc and even well beyond. Kinematical distances in this region are very unreliable for distances up to $\sim 4 \mathrm{kpc}$ because of the nearzero radial velocity gradient. For the objects studied in this paper, however, we do not expect a significant confusion because all spatially resolved objects clearly point towards Cyg OB2 and are thus shaped by the radiation of the stars.

Using the observational Herschel FIR-data (Sect. 2) and the Herschel derived column density, temperature, and farultraviolet (FUV) field maps (Sects. 3 and 4), we classify the various features seen in the data (Sect. 5), calculate their lifetimes, and discuss their possible evolution (Sect. 6). A comparison of the pre- and protostellar sources found within various structures will be made in a subsequent paper.

\section{Observations, dust column density, and dust temperature maps}

Two fields covering the Cygnus $\mathrm{X}$ region were observed with the Herschel satellite (Pilbratt et al. 2010) in parallel mode. We present Herschel imaging observations at $70 \mu \mathrm{m}$ and $160 \mu \mathrm{m}$ from Photoconductor Array Camera and Spectrometer (PACS; Poglitch et al. 2010), and at $250 \mu \mathrm{m}, 350 \mu \mathrm{m}$, and $500 \mu \mathrm{m}$ from Spectral and Photometric Imaging Receiver (SPIRE; Griffin et al. 2010). Cygnus X South was observed on May 24, 2010 (obsIDs 1342196917 and 1342196918 for the nominal and orthogonal directions, respectively) and Cygnus X North on December 18, 2010 (obsIDs 13422211307 and 1342211308). This paper uses the observations of a part of both regions. The DR21 filament in Cygnus X North has already been presented in Hennemann et al. (2012, Herschel imaging) and White et al. (2010, Herschel spectroscopy). For this paper, we employed a more recent Herschel interactice processing environment (HIPE) version (10.0.2751) for the PACS and SPIRE data reduction in which we used modified pipeline scripts. Data collected during the turnaround of the satellite were included to insure a larger area was covered by the observations. The resulting Level1 contexts for each scan direction were combined using the "naive" map maker in the destriper module. The conversion of the maps into surface brightness (from Jy/beam into $\mathrm{MJy} / \mathrm{sr}$ ) was made 


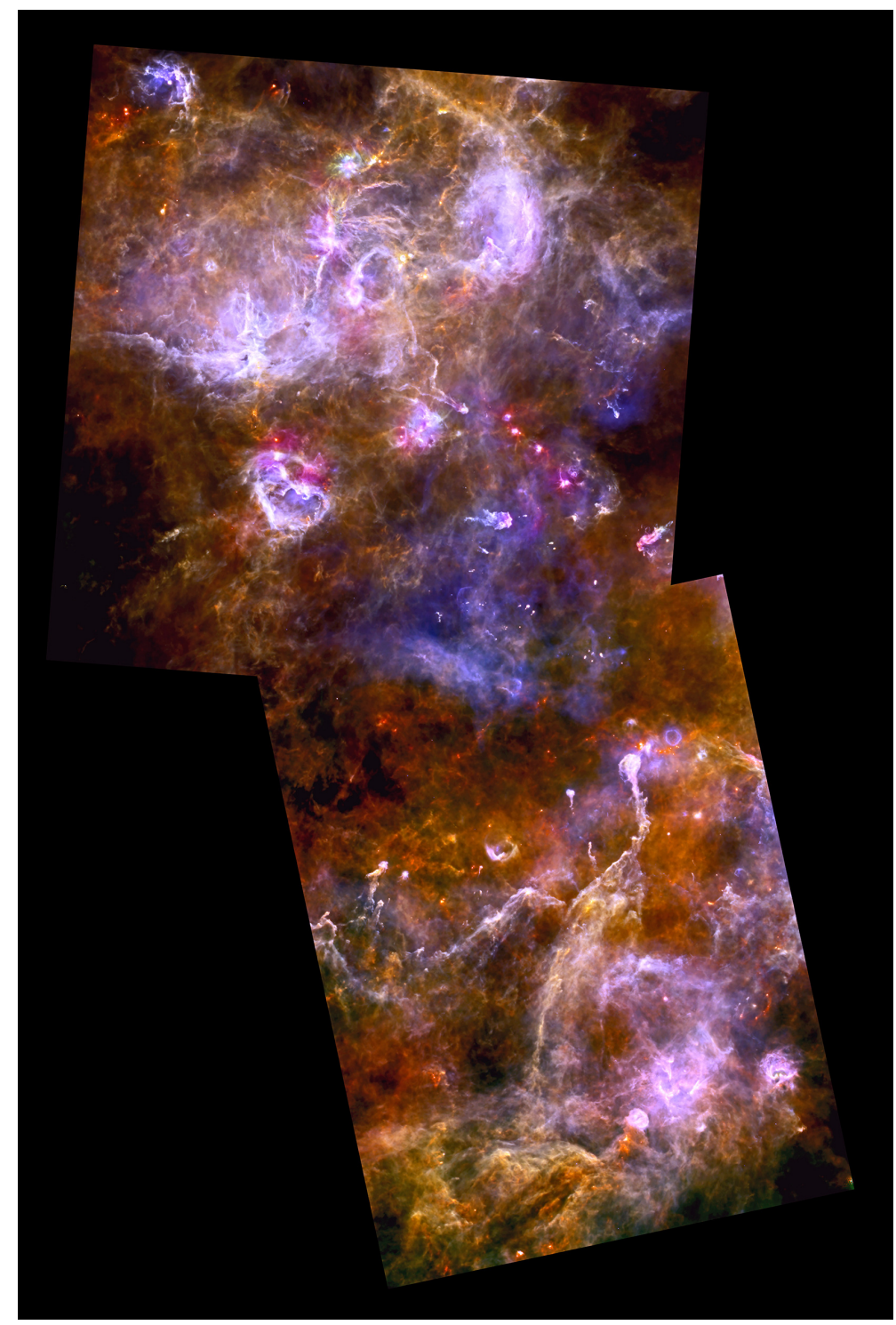

Fig. 1. Three-colour image (blue $=70 \mu \mathrm{m}$, green $=160 \mu \mathrm{m}$, red $=500 \mu \mathrm{m}$, all maps are absolutely calibrated) of the Cygnus $\mathrm{X}$ complex (credit: ESA/PACS/SPIRE) oriented in RA/Dec, i.e., north to the top, east to the left. The Cyg OB2 association is located slightly above the center of the figure. The image covers a range of $\mathrm{RA}(2000) \sim 20^{\mathrm{h}} 30^{\mathrm{m}}$ to $20^{\mathrm{h}} 45^{\mathrm{m}}$ and $\operatorname{Dec}(2000) \sim 38^{\circ}$ and $43^{\circ}$. Blue colours indicate gas heated by highmass stars of the cluster and white colours indicate smaller H II regions that locally heat the gas and create bubble-like features. Colder extended emission mainly arising from the molecular cloud is shown in red. Numerous pillars and globules, in particular in the southern part of Cygnus X, point towards Cyg OB2.

using the beam-areas obtained from measurements of Neptune (March 2013, see SPIRE handbook). The map offsets of the Level2 data were then determined using Planck and IRAS observations (Bernard et al. 2010). The zero levels for the SPIRE $250 \mu \mathrm{m}, 350 \mu \mathrm{m}$, and $500 \mu \mathrm{m}$ data were also determined using the ZEROPOINTCORRECTION task in HIPE and found to be consistent with offsets provided by J.-P. Bernard. PACS data were reduced up to Level1 using HIPE 10.0.2751 and then v. 20 of the Scanamorphos software package which performs baseline and drift removal before regridding (Roussel 2013). See Hennemann et al. (2012), Hill et al. (2011) and Könyves et al. (2015) for further details. The angular resolutions at $70 \mu \mathrm{m}, 160 \mu \mathrm{m}, 250 \mu \mathrm{m}$, $350 \mu \mathrm{m}$, and $500 \mu \mathrm{m}$, are $\sim 6^{\prime \prime}, \sim 12^{\prime \prime}, \sim 18^{\prime \prime}, \sim 25^{\prime \prime}$, and $\sim 36^{\prime \prime}$, respectively.

Figure 1 shows a three-colour image of the whole Cygnus $\mathrm{X}$ region observed as part of the HOBYS program. The picture reveals impressively the way in which bright star-forming regions, emitting mainly at $70 \mu \mathrm{m}$ and $160 \mu \mathrm{m}$ (blue/green), are nestled in the larger cloud which is pervaded by a web of filaments. Colder gas is well traced by dust emission at $500 \mu \mathrm{m}$ (red). At the very center, the diffuse blue emission of PACS at $70 \mu \mathrm{m}$ indicates heating by the Cyg OB2 association (see Fig. 2 to locate the highest-mass stars). The $\mathrm{H}$ II regions of Cygnus X (DR18, 20 , and 22 in the northern region and DR15 in the southern region) stand out as bright emission regions in the three-colour image. The majority of pillars and globules in the figure, however, point towards the center of Cyg OB2, the most important source of UV-illumination (see Sect. 3). A zoom into the vicinity of Cyg OB2 is shown by a PACS image at $70 \mu \mathrm{m}$ (Fig. 2); individual maps at $70 \mu \mathrm{m}$ to $500 \mu \mathrm{m}$ of these cutouts are shown in Appendix A. In Sects. 5 and 6, we focus on the regions indicated in this plot and discuss the properties of the observed features.

Column density and dust temperature maps at an angular resolution of 36" (all maps were smoothed to this lowest resolution 


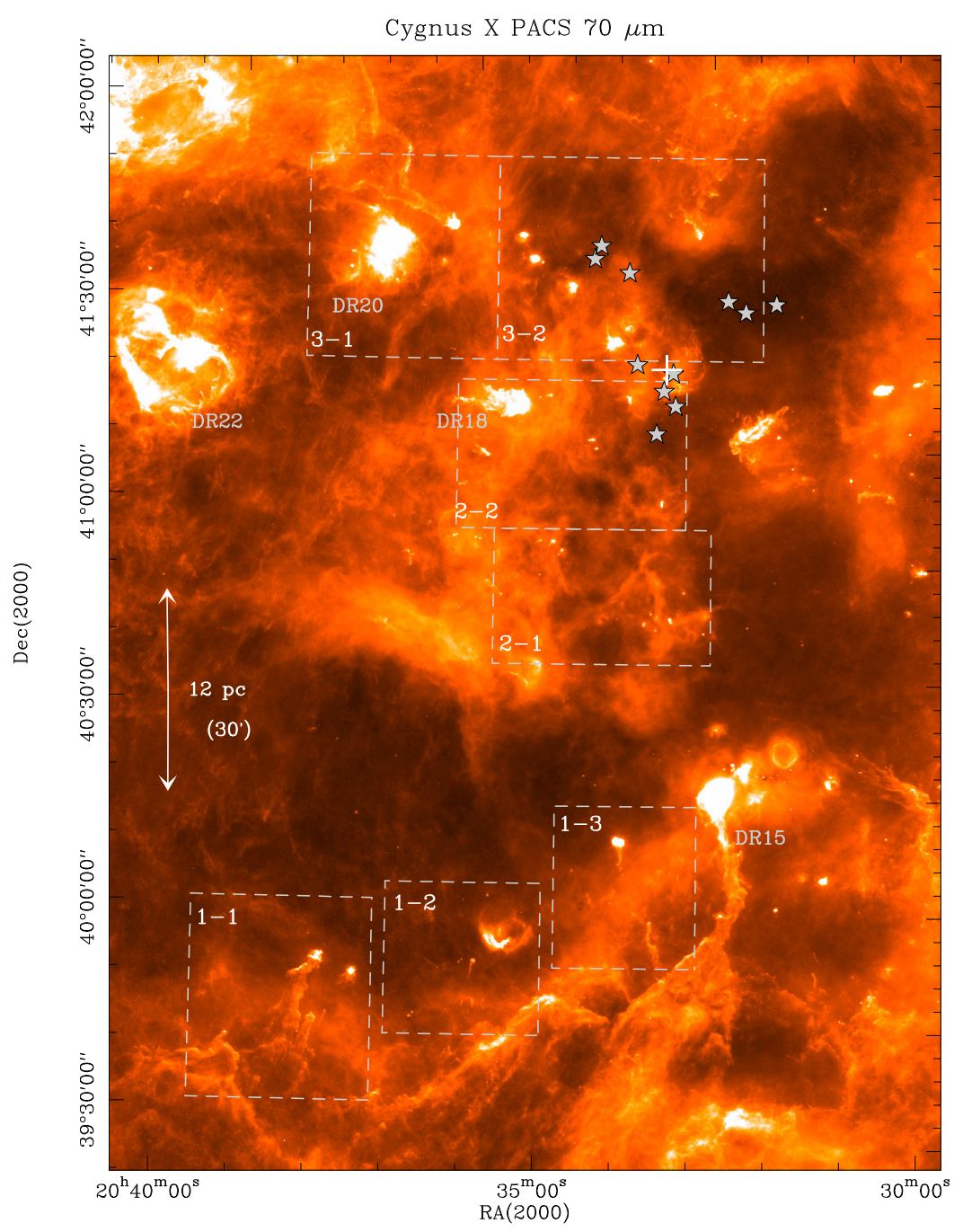

Fig. 2. Herschel $70 \mu \mathrm{m}$ PACS image (0 to $1000 \mathrm{MJy} / \mathrm{sr}$ ) of the central region around Cyg OB2 (the most massive stars of the cluster are indicated by gray stars). The cross indicates the position of star Cyg OB2 \#8 at RA $(2000)=20^{\mathrm{h}} 33^{\mathrm{m}} 16^{\mathrm{s}}$ and $\operatorname{Dec}(2000)=41^{\circ} 18^{\prime} 45^{\prime \prime}$, frequently used to define the center of Cyg OB2 (e.g., Wright et al. 2015). The H II regions DR15, 18, 20, and 22 are labelled and the regions shown and discussed in more detail are outlined with dashed lines and numbered.

of the $500 \mu \mathrm{m}$ map) were made with a pixel-by-pixel SED (spectral energy distribution) fit from $160 \mu \mathrm{m}$ to $500 \mu \mathrm{m}$, as described in, for example, Hill et al. (2011). For the SED fit, we used a specific dust opacity per unit mass (dust and gas) approximated by the power law $\kappa_{\lambda}=0.1(\lambda / 300 \mu \mathrm{m})^{\beta} \mathrm{cm}^{2} \mathrm{~g}^{-1}$ with $\beta=2$ (Roy et al. 2014), and left the dust temperature and column density as free parameters. We checked the SED fit of each pixel and determined from the fitted surface density the $\mathrm{H}_{2}$ column density. The fit assumes a constant temperature for each pixel along the line of sight, an assumption that is not fullfilled in regions with strong temperature gradients. A detailed study of the dust properties in Orion A (Roy et al. 2013), however, showed that the single temperature model provides a reasonable fit, but that the dust opacity varies with column density by up to a factor of two. We thus estimate that the final uncertainties of the column density map are between $20 \%$ and $30 \%$.

\section{The FUV-field in Cygnus $X$}

Cyg OB2 is one of the largest and most massive OB associations in the Galaxy with a mass of around $3 \times 10^{4} M_{\odot}$ (Drew et al. 2008) at a distance of only $1.4 \mathrm{kpc}$ (Rygl et al. 2012 from parallax observations). It is the richest aggregate in the Cygnus $\mathrm{X}$ region (e.g., Hanson 2003, Comerón \& Pasquali 2012), though the whole Cygnus $\mathrm{X}$ region contains several OB-associations, and is clearly the most important source of FUV-impact for the features we study here. A recent inventory of the central massive stars in Cyg OB2 compiles 52 O-stars and 3 Wolf-Rayet stars (Wright et al. 2015). They are the most important sources of feedback (radiation, winds) though the whole Cyg OB2 association is more extended (Comerón et al. 2008; Comerón \& Pasquali 2012). We evaluate the FUV-field expressed as a Habing field ${ }^{2}$, produced by the $52 \mathrm{O}$-stars listed in Wright et al. (2015). The ionising fluxes (Wright et al., priv. comm.) were calculated taking into account not just their spectral types, but also their exact luminosities, and binary companions. The most recent stellar atmosphere models were used, including the revised effective temperature scale in Martins et al. (2005), and the luminosity shift that this leads to. We assume a simple $1 / r^{2}$ decrease of the flux and project all stars in the plane of the sky, ignoring possible attenuation by diffuse gas and blocking by molecular clumps. Additional sources of illumination, for example the

2 With $G_{0}$ expressed in units of the Habing field (Habing 1968) $2.7 \times$ $10^{-3} \mathrm{erg} \mathrm{cm}^{-2} \mathrm{~s}^{-1}$ and the relation $G_{0}=1.7 \chi$ with the Draine field $\chi$. 

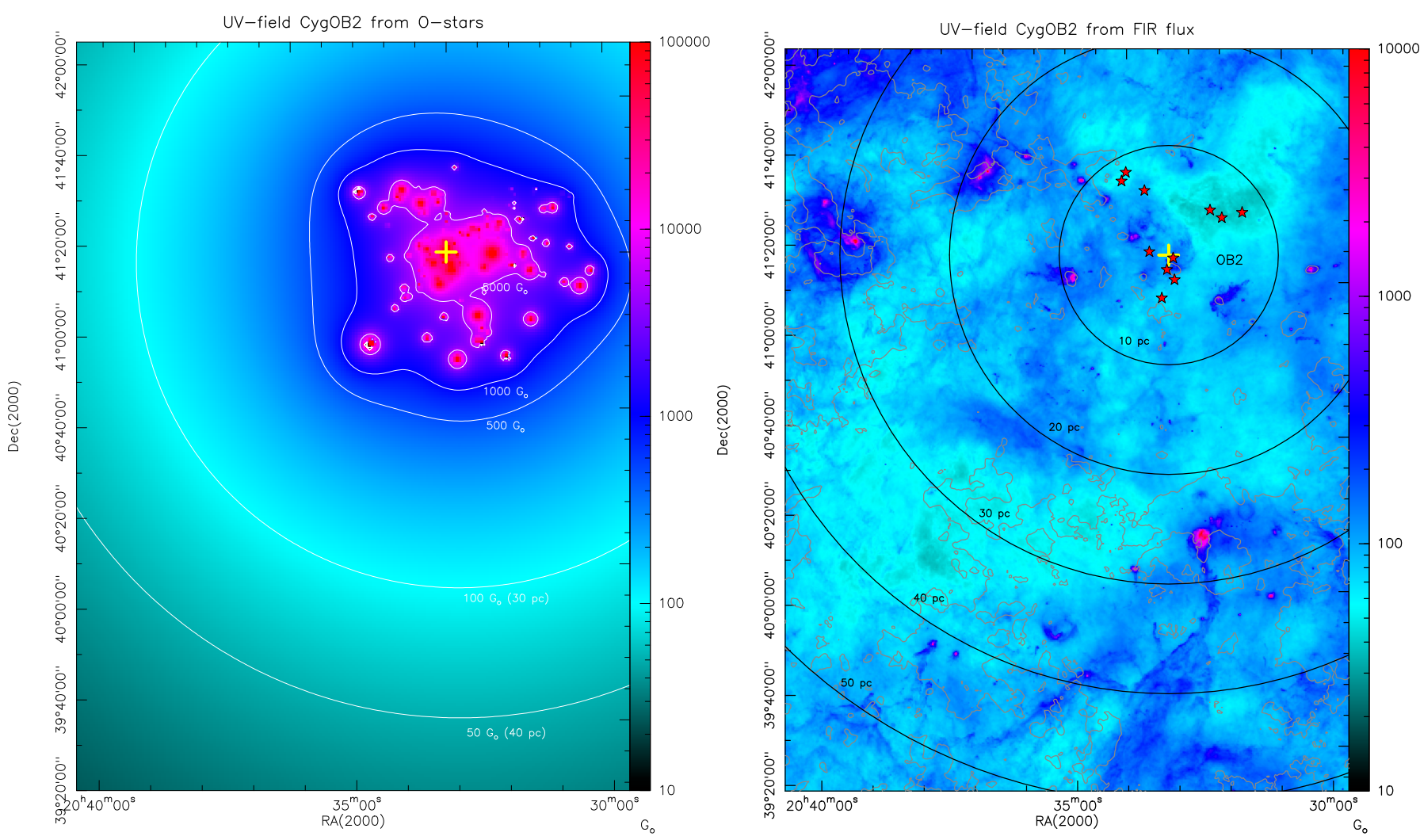

Fig. 3. Left: UV-field in units of Habing $\left(G_{0}\right)$ determined from a census of all O-stars given in Wright et al. (2015). Right: UV-field in units of Habing $\left(G_{0}\right)$ for the central region around Cyg OB2 (limits as in Fig. 2) determined from the Herschel $70 \mu \mathrm{m}$ and $160 \mu \mathrm{m}$ fluxes. This image shows the impinging radiation. The most luminous O-stars of Cyg OB2 (Wright et al. 2015) are indicated as red stars and the yellow cross indicates the approximate center of the association. Concentric circles indicate the projected distance to this center in the plane of the sky, assuming a distance of $1.4 \mathrm{kpc}$ to Cyg OB2. Gray contours $\left(2 \mathrm{~K} \mathrm{kms}^{-1}\right.$ to $22 \mathrm{~K} \mathrm{kms}^{-1}$ in steps of $\left.2 \mathrm{~K} \mathrm{kms}^{-1}\right)$ display line integrated $\left(-10\right.$ to $\left.20 \mathrm{~km} \mathrm{~s}^{-1}\right){ }^{13} \mathrm{CO}^{1} \rightarrow 0$ emission at a resolution of 45" (Schneider et al. 2011).

more widespread $\mathrm{O}$ - and B-star population of Cyg OB2, are also not considered in this method. The resulting FUV-field is shown in Fig. 3 (left panel). Obviously, the field is strongest in the immediate environment of the stars, that is, the central $10 \mathrm{pc}$ circle, reaching values of up to a few $10^{5} G_{0}$ and drops to $\sim 50 G_{0}$ in 40 pc distance.

In a second approach, we estimate the FUV-field from the total FIR intensity $\left(I_{\mathrm{FIR}}\right)$, assuming that the radiation from the massive stars heating the dust is re-radiated mainly at FIR wavelengths. With Herschel, we evaluate the FUV-field by adding the intensities at $70 \mu \mathrm{m}$ and $160 \mu \mathrm{m}$ (at an angular resolution of $12^{\prime \prime}$ ) to $I_{\mathrm{FIR}}\left[10^{-17} \mathrm{erg} \mathrm{cm}^{-2} \mathrm{~s}^{-1} \mathrm{sr}^{-1}\right]$ (see Kramer et al. 2008; Roccatagliata et al. 2013 for the methodology):

$$
F_{\text {fuv }}\left[G_{0}\right]=\left(4 \pi I_{\text {FIR }} 1000\right) / 1.6 .
$$

Note that the use of $160 \mu \mathrm{m}$ emission as a tracer of the FUV-field can be disputable in regions dominated by cooler gas (which is not the case for Cygnus X) because flux at this wavelength can also come from cold thermal dust emission from the molecular cloud (the wavelength range $160 \mu \mathrm{m}$ to $500 \mu \mathrm{m}$ is used for the SED fit to determine the column density). For Cygnus X, the $160 \mu$ m emission contributed approximately $20 \%$ to the total UV-field, displayed in Fig. 3 (right), that ranges between a few hundred $G_{0}$ to more than $10^{4} G_{0}$.

This method emphasizes the PDR surfaces that stand out prominently. These are illuminated by a UV-field of at least a few hundred $G_{0}$. Internal $\mathrm{H}_{\text {II }}$ regions can also contribute to the local FUV-field as in the case of the bright globule (g1) in
Cygnus X (see below) that contains early B-stars. Here, the average UV-field across the globule head is $550 G_{0}$ (see Table 1), but increases up to $7500 G_{0}$ in a $12^{\prime \prime}$ beam at the position of the B-star. Note, however, that the UV-field can only be calculated on the surfaces of molecular condensations (clumps, filaments, pillars, globules, etc.). In the ionized phase, the UV-field is, of course, present but can not be evaluated with this method. Instead, the field is estimated as outlined above, that is, from the photon flux of the stars (Fig. 3, right). The maps are thus not comparable but complementary, and a combination of both maps probably represents the overall FUV-field in Cygnus X best.

\section{Identification of objects}

From the five Herschel wavelength bands, the $70 \mu \mathrm{m}$ map (Fig. 2 and Appendix A) is the best suited to trace UV-illuminated features because the emission at $70 \mu \mathrm{m}$ arises from warm dust and has the highest angular resolution $\left(6^{\prime \prime}\right)$. The maps at shorter wavelengths $(70 \mu \mathrm{m}, 160 \mu \mathrm{m}$, and $250 \mu \mathrm{m})$ all start at $0 \mathrm{MJy} / \mathrm{sr}$ and show that with increasing wavelength, the amount of emission from colder dust increases and is visible as a diffuse background.

The $70 \mu \mathrm{m}$ maps are dominated by emission from UV-heated dust, and so pillars, globules, and other features clearly stand out from a lower background, as is indicated by the contour at $400 \mathrm{MJy} / \mathrm{sr}$ (i.e., the sharp transition from red to green in the map). We thus used this contour to trace UV-illuminated features in the $70 \mu \mathrm{m}$ maps and not the maps at longer wavelengths that 

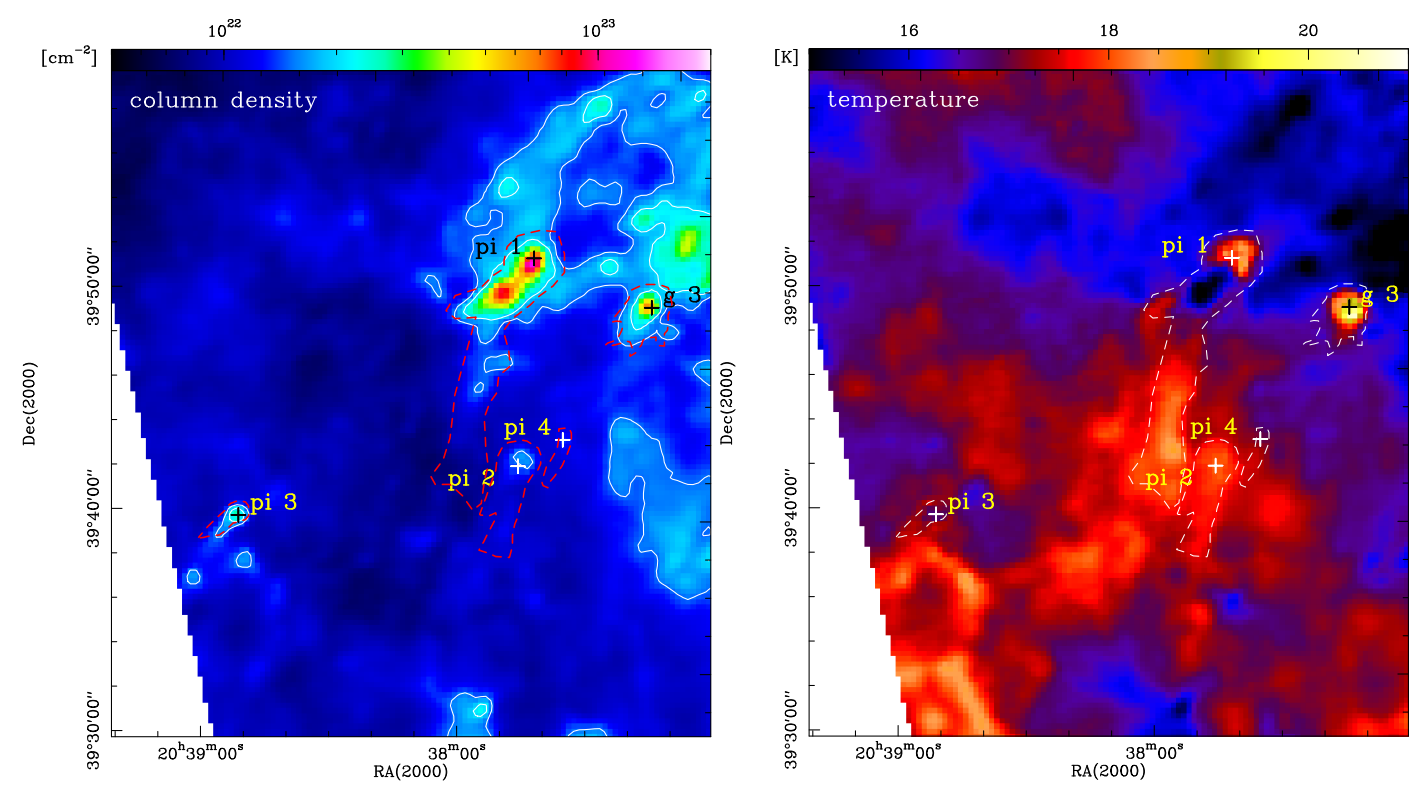

Fig. 4. Region 1-1: column density (left) and dust temperature (right). Pillars are indicated by "pi", globules by "g", proplyds/proplyd-like by "pr", condensations by "c", and EGGs by "e". The red dashed (white dashed in the right panel) contours outline the source shape based on the $70 \mu \mathrm{m}$ map (derived from the $400 \mathrm{MJy} / \mathrm{sr}$ threshold). The column density contours correspond to the levels 1.5 and $2.0 \times 10^{22} \mathrm{~cm}^{-2}$.

are dominated by cold dust, mixed with mostly molecular gas. For display reasons, we set the lower limits to $150 \mathrm{MJy} / \mathrm{sr}$ and $70 \mathrm{MJy} / \mathrm{sr}$ for the $350 \mu \mathrm{m}$ and $500 \mu \mathrm{m}$ maps, respectively. The $500 \mu \mathrm{m}$ maps thus show only the densest parts of the objects, that is, the heads of pillars or globules which are also traced in the column density maps. It is only with the temperature maps, that it is possible to distinguish whether the gas is warm or cold.

For display reasons, we divided the map shown in Fig. 2 into subregions covering many observed features. The resulting maps reveal many objects with different shapes and sizes in the vicinity of the Cyg OB2 cluster. As explained above, we take the contour level $400 \mathrm{MJy} / \mathrm{sr}$ (shown in the individual $70 \mu \mathrm{m}$ maps of each subregion; see Appendix A) as a threshold to define the borders of all objects. This contour typically corresponds to a column density of $1-2 \sim 10^{22} \mathrm{~cm}^{-2}$. We note, however, that the shape of the objects defined in the $70 \mu \mathrm{m}$ maps do not always correspond one-to-one to the column density contour. For example, clumps within molecular clouds are prominent column density peaks but do not show up in the $70 \mu \mathrm{m}$ map. We classified elongated, column-like structures that are still attached to the molecular cloud as pillars, isolated head-tail features as globules when they are large (typically $>1^{\prime}$ ) and prominent and as EGGs when they are small $\left(<1^{\prime}\right)$ and faint. Centrally condensed objects without tails were called condensations. The term proplydlike was used for the sources given in Wright et al. (2012) as well as to some new ones, which probably fit into this scheme, detected in the Herschel images. All other features in the map that we did not classify have a more or less arbitrary shape and are most often bright edges of $\mathrm{H}_{\text {II }}$ regions. Our classification is complete, within the observed area, though we do not cover the whole Cygnus $\mathrm{X}$ region. Our main objective, however, is to derive typical values and discuss differences between the features representing different object classes in the various subregions.

The contours and labels of the objects identified in this way are shown in the column density and temperature maps of each subregion (see Figs. 4 to 10). Obviously, for some sources, the shape outlined by the $70 \mu \mathrm{m}$ contour does not fully correspond to its shape in the column density or temperature map. For example,
Pillar 1 in region $1-1$ (Fig. 4) consists of a dense head with two peaks in column density of which one is cold $(\sim 14 \mathrm{~K})$ and the other warm $(\sim 19 \mathrm{~K})$, while the base of the pillar does not show up in the column density map. With this caveat in mind, we then determine the physical properties (column density, temperature, density, mass, length and width or radius, etc.) of the objects (Table 1 lists these quantities). In the following sections, we qualitatively describe the maps and objects and quantitatively discuss their various properties.

\section{Results}

\subsection{Description of maps}

Regions 1-1, 1-2, and 1-3 (Figs. 4-6) contain five pillars and two globules and some examples of EGGs (five in total gathered in a "swarm", named e1 to e5), best visible in the $70 \mu \mathrm{m}$ Herschel map in the Appendix. They have the same orientation toward north as globule g2 suggesting that they are being influenced by Cyg OB2. The condensations (c1 to c3) in subregion 1-2 are also faint but are more spherical and show up mainly at $500 \mu \mathrm{m}$.

Regions 2-1 and 2-2 (Figs. 7, 8) contain the ten Cyg OB2 proplyd-like objects reported by Wright et al. (2012) that were detected in $\mathrm{H} \alpha$ and Spitzer images. Two sources look spherical (pr1, pr8) in the Herschel images, even in the highest angular resolution $70 \mu \mathrm{m}$ map, and can thus be partly unresolved, while the others have more complex head/tail shapes with bright ionization fronts that are oriented towards the center of Cyg OB2. However, all proplyd-like objects show an elongated structure pointing toward the center of Cyg OB2 in high resolution optical and near-IR images (Wright et al. 2012). Close to proplyd \#5 (Wright et al. 2012), we detected some additional faint features that we name pr5a-d. The size scale of all these objects is typically around $0.1 \mathrm{pc}$, in agreement with what Wright et al. found. These authors are not certain about the nature of these features, suggesting that they are photo-evaporating protostars and naming them proplyd-like objects. Guarcello et al. (2013) 

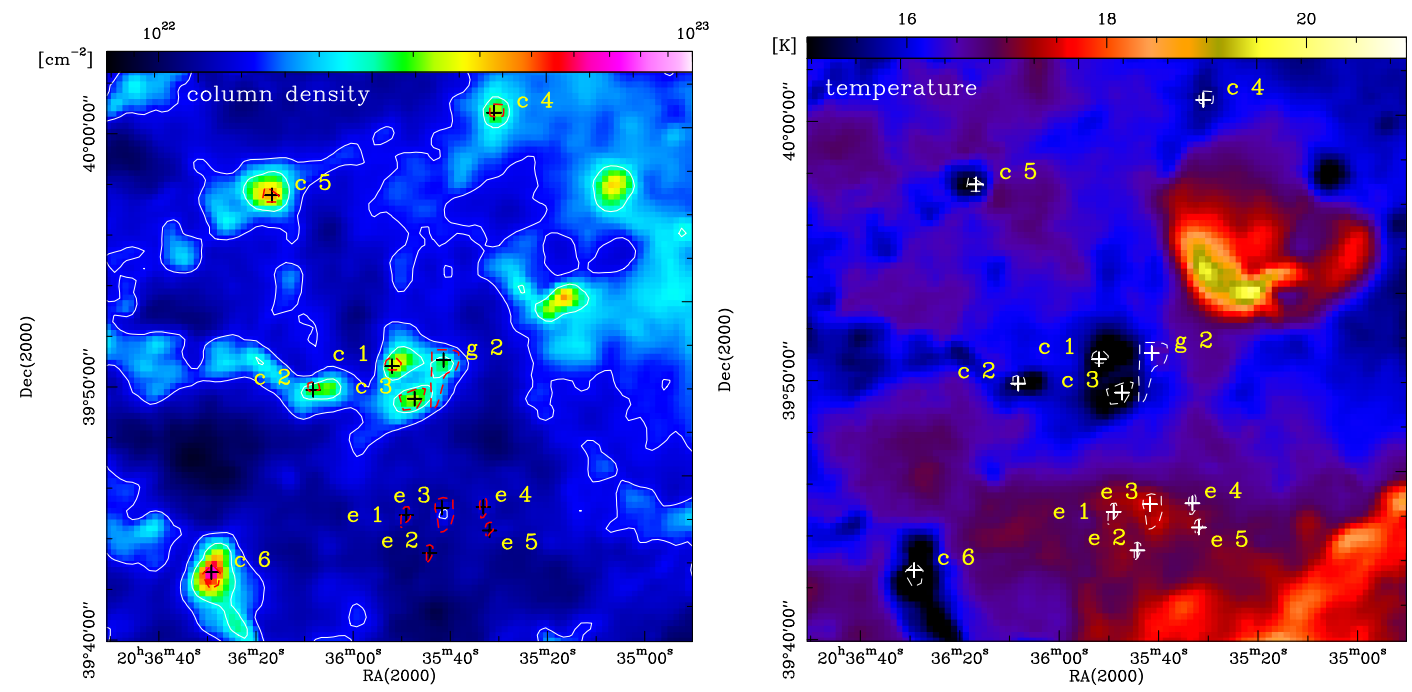

Fig. 5. Region 1-2: as in Fig. 4. The column density contours correspond to the levels 1.5 and $2.3 \times 10^{22} \mathrm{~cm}^{-2}$.
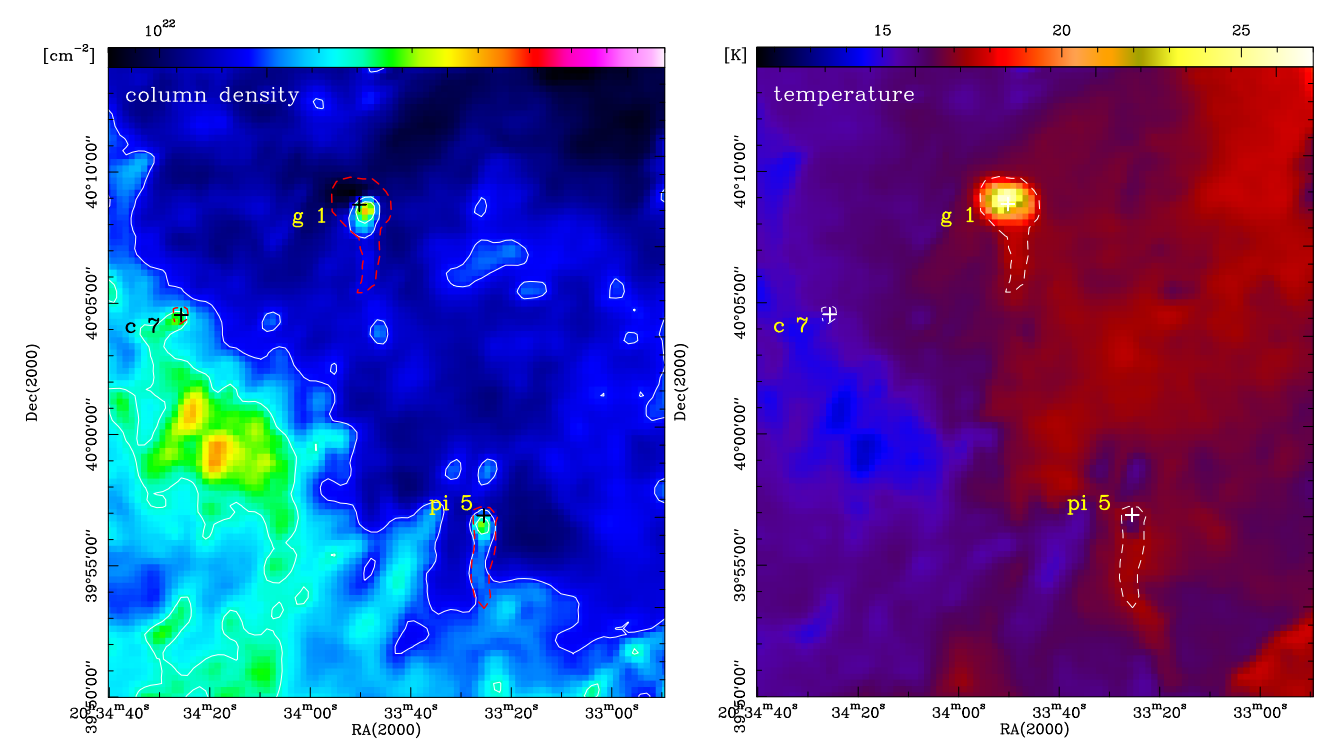

Fig. 6. Region 1-3: as in Fig. 4. The column density contours correspond to the levels 1.5 and $2.3 \times 10^{22} \mathrm{~cm}^{-2}$.

classified some of them as embedded stars with disks, and a recent spectroscopic study (Guarcello et al. 2014) showed that at least \#5 and \#7 are actively accreting protostars and that the disk of \#7 is photoevaporating. Globule $\mathrm{g} 4$ coincides with the $\mathrm{H}$ II region DR18, which hosts a strong IR-source (IRAS 20343+4129, MSX6G080.3624+00.4213) at near-, mid-, and far-IR wavelengths. Comerón \& Torra (1999) observed DR18 at IR and optical wavelengths and found an arc-shaped nebula, externally illuminated by a nearby B0.5V star. The globular shape is most likely caused by the object's proximity to the Cyg OB2 cluster (see Fig. 2), though this source is not a typical example of a globule (like g1) because it is significantly more massive and extended.

Region 3-1 (Fig. 9) corresponds to the star-forming region DR20 (e.g., Odenwald et al. 1990) and includes one of the largest and most prominent pillars (pi6) in the Cyg OB2 environment. The pillar clearly points towards the center of OB2.
Region 3-2 (Fig. 10) contains another pillar (pi7) and three cold, irregularly shaped gas clumps. These clumps are probably not influenced by the close-by Cyg OB2 cluster because they have no preferred direction, in contrast to pi 7 which points clearly towards the cluster center. Although projection effects can not be excluded, it is more likely that the clumps are massive structures still embedded in a more extended molecular cloud, and which are probably forming small clusters because we observe internal heating (see temperature map).

\subsection{Physical properties}

Table 1 gives an overview of the column density $N\left(\mathrm{H}_{2}\right)$, mass $M$, density $n$, temperature $T$, equivalent radius $r$, length $l$ and width $w$ for the elongated features, surface density $\Sigma$, and UV-flux $F_{\text {fuv }}$ (derived from the Herschel data), we determined for each object from the Herschel observations. The length and width of the pillars were directly measured on the $70 \mu \mathrm{m}$ map. The column density maps were used to calculate average column density, 

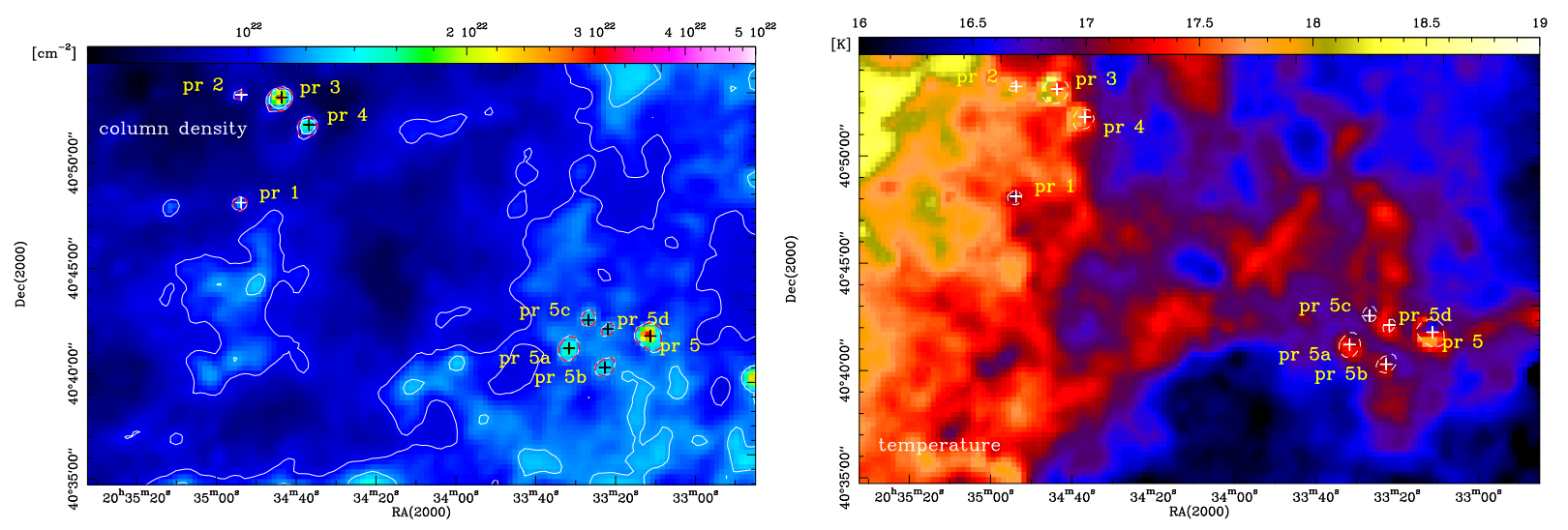

Fig. 7. Region 2-1: as in Fig. 4. The column density contours correspond to the levels 1.0 and $1.2 \times 10^{22} \mathrm{~cm}^{-2}$.
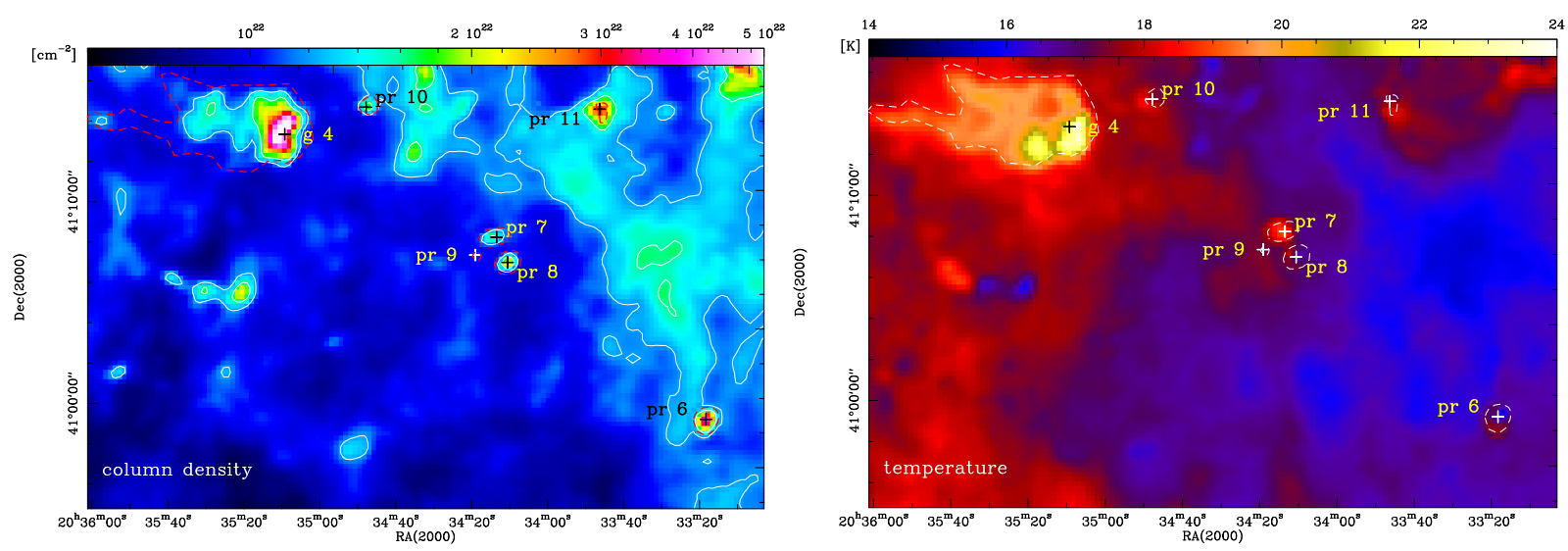

Fig. 8. Region 2-2: as in Fig. 4. The column density contours correspond to the levels 1.2 and $1.6 \times 10^{22} \mathrm{~cm}^{-2}$.

density, and mass within the areas defined by the $70 \mu \mathrm{m}$ contour at $400 \mathrm{MJy} / \mathrm{sr}$ that we used to identify different features (see Sect. 4). Mass was determined following Eq. (2), using the column density $N\left(\mathrm{H}_{2}\right)$ in $\mathrm{cm}^{-2}$, distance $d$ in parsec, and area $A$ in square degrees (see, e.g., Schneider et al. 1998)

$$
M=6.6 \times 10^{-24} N\left(H_{2}\right) d^{2} A .
$$

We estimate that the column density, mass, and density values are correct to within a factor of $\sim 2$. The uncertainties mainly arise from the assumed dust opacity, and the possible variation in temperature along the line-of-sight not accounted for in the SED fitting. We consider the error on the distance to be low because the distance of Cyg OB2 has been very accurately determined through parallax (Rygl et al. 2012). Since all objects we discuss here are directly affected by this cluster they must be at more or less the same distance. The minimum, maximum and average temperatures given in the table were derived from the temperature maps. Since proplyd-like objects, EGGs, and condensations were barely resolved, minimum and maximum values were omitted. We did not include any irregular features in the table because they do not form an individual class of objects, but are mostly $\mathrm{H}$ II regions. The last line in each source category lists the average (mean) value. The main results extracted from the table are:

\section{Size and density}

EGGs, proplyd-like objects, and condensations are small (typically $0.1 \mathrm{pc}$ to $0.25 \mathrm{pc}$ ). Their average densities are $2 \times 10^{4} \mathrm{~cm}^{-3}$,
$1.5 \times 10^{4} \mathrm{~cm}^{-3}$, and $5.5 \times 10^{4} \mathrm{~cm}^{-3}$, respectively. Comparing them to general molecular cloud features, condensations correspond to the dense cores typically found in submm-continuum imaging of high-mass star-forming regions (e.g., Motte et al. 2007 for the Cygnus X region), but they are significantly larger and more massive than the dense cores found with Herschel in the nearby clouds of the Gould Belt (e.g., Könyves et al. 2015). The proplyd-like objects are much larger than the ones found in Orion A ( $\sim$ a factor of 10, O'Dell et al. 1993), and also larger than those detected in the Carina nebula ( $\sim$ a factor of 2, Smith et al. 2003). Pillars and globules show a density gradient from high values $n>10^{4} \mathrm{~cm}^{-3}$ for the head, and $n<10^{4} \mathrm{~cm}^{-3}$ for the tail. The length of pillars varies between $0.6 \mathrm{pc}$ and $3 \mathrm{pc}$.

\section{Temperature}

The temperature range of all objects is between $14 \mathrm{~K}$ and $26 \mathrm{~K}$ (average temperatures). The lowest temperatures are found for condensations $(\langle T\rangle \sim 15 \mathrm{~K})$, and highest for globules $(\langle T\rangle \sim 18 \mathrm{~K})$. All other objects typically have a temperature around $17 \mathrm{~K}$. Pillars and globules show a temperature gradient from their warm UV-heated (external and possibly internal) head to a colder tail.

Mass

The highest mass objects (typically a few hundred $M_{\odot}$ ) are found amongst pillars and globules, mainly due to their large extents (e.g., equivalent radii larger than $0.5 \mathrm{pc}$ ) since their average densities $\left(\sim 5000 \mathrm{~cm}^{-3}\right)$ are low compared to the other 

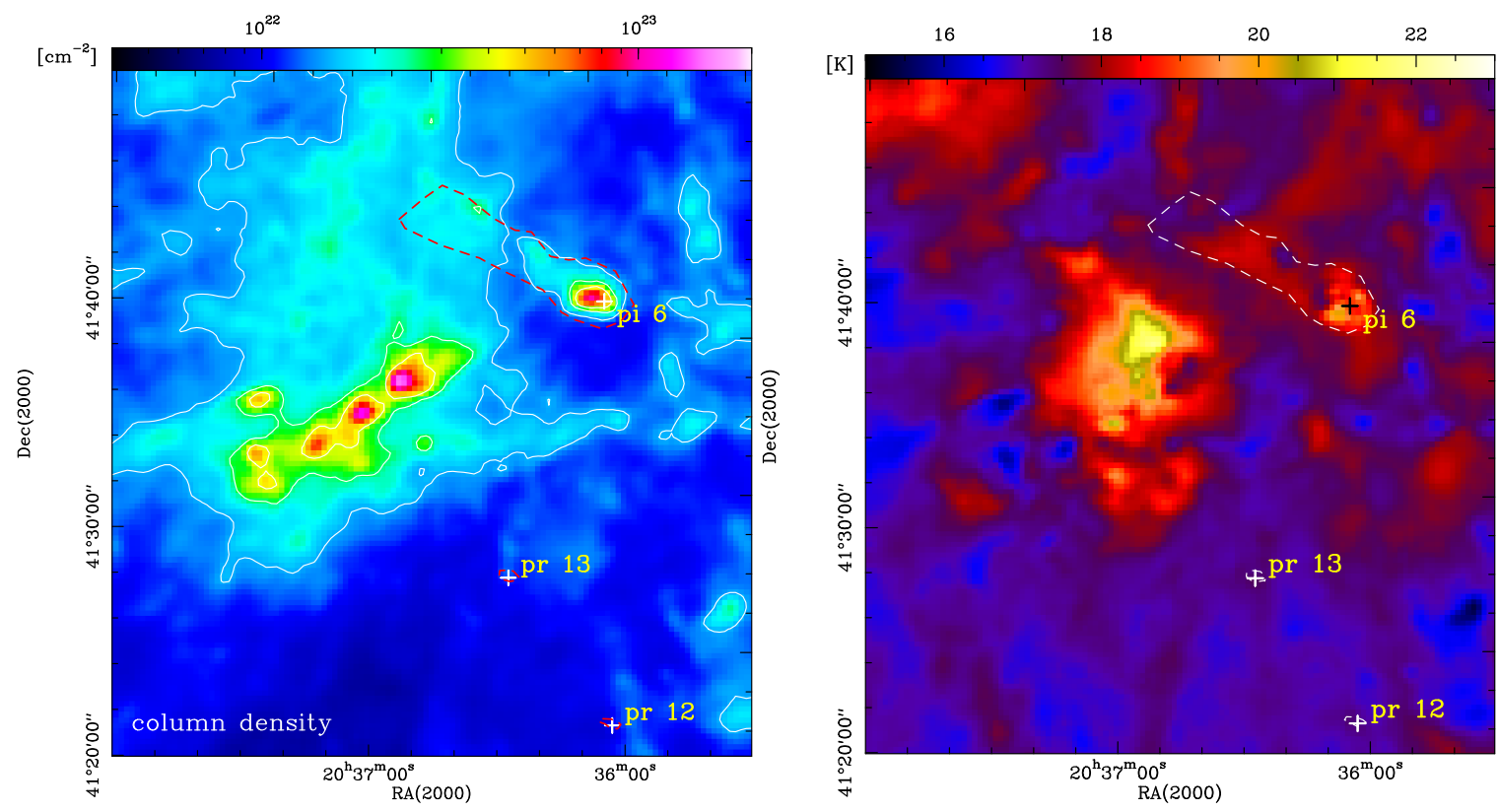

Fig. 9. Region 3-1: as in Fig. 4. The column density contours correspond to the levels 1.5 and $4.0 \times 10^{22} \mathrm{~cm}^{-2}$.
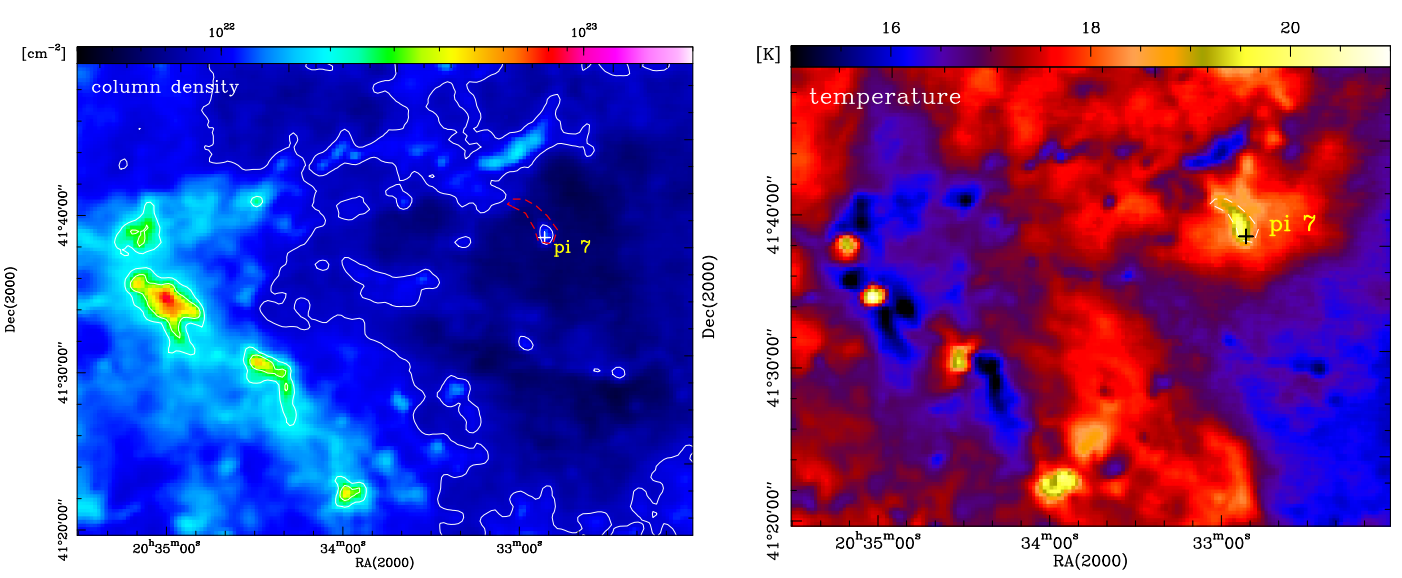

Fig. 10. Region 3-2: as in Fig. 4. The column density contours correspond to the levels 1.0 and $1.7 \times 10^{22} \mathrm{~cm}^{-2}$.

features. We note, however, that the mass range can be quite varied, for example $\sim 50 M_{\odot}$ for pi 4 but $\sim 1700 M_{\odot}$ for pi 1 . Their physical properties thus correspond to those typically derived for clumps inside molecular clouds (e.g., Schneider et al. 2006, Lo et al. 2009). Condensations and proplyd-like objects have masses of typically several tens of $M_{\odot}$ and EGGs have masses lower than $10 M_{\odot}$. All objects are thus potential star-forming sites with large enough gas reservoirs to be so.

\section{Surface density}

Surface densities are highest for condensations and globules, i.e., $\sim 660 M_{\odot} / \mathrm{pc}^{2}$ and $\sim 380 M_{\odot} / \mathrm{pc}^{2}$, respectively. All other objects have $\langle\Sigma\rangle$ around $240-280 M_{\odot} / \mathrm{pc}^{2}$.

\section{$U V$-flux}

The UV-flux does not reflect an internal property of the detected features, it depends on its location and if it is only externally illuminated or contains an internal source. Obviously, high implied UV fluxes are found for all objects (mainly the proplyd-like objects), close to the central Cyg OB2 cluster. Two objects, globules g1 and g4, presumably have internal heating sources which explains their rather high implied average UV fluxes $\left(549 G_{0}\right.$ and $\left.428 G_{0}\right)$, respectively.

\section{Analysis and discussion}

The original "radiative driven implosion" scenario (Bertoldi 1989; Lefloch \& Lazareff 1994; Miao et al. 2009) for the formation of pillars and globules involves UV-radiation illuminating a pre-existing clumpy molecular cloud and photoevaporating the lower density gas, leaving only the densest cores, which may collapse to form stars. Though recent hydrodynamic simulations including radiation (Gritschneder et al. 2009, 2010; Tremblin et al. 2012a,b) successfully model pillars and globules emphasizing the importance of turbulence, we will focus here on studying the lifetime of UV-illuminated features using the more classical approach.

\subsection{Lifetimes}

The model for photoevaporation of dense clumps we use here is based on that of Johnstone et al. (1998). It calculates the mass 
loss rate $\dot{M}$ considering the external photon flux $\phi_{49}$, the number of ionizing photons from O-stars per second (in units of $10^{49}$ ), that impinges on a clump with radius $r_{14}$ (in units of $10^{14} \mathrm{~cm}$ ) at the distance to the central source $d_{17}$ (in units of $10^{17} \mathrm{~cm}$ ):

$$
\dot{M}=9.5 \times 10^{-9} \phi_{49}^{1 / 2} r_{14}^{3 / 2} d_{17}^{-1}\left[M_{\odot} / \mathrm{yr}\right] \text {. }
$$

For the photon flux, we adopted as a lower limit the total flux from the most luminous O-stars clearly identified as members of Cyg OB2 in Hanson (2003) and Wright et al. (2015), and ignored possible extinction. The total photon flux of $10^{50.3} \mathrm{~s}^{-1}$ was determined by their spectral type using the published values given in Sternberg et al. (2003). Assuming a constant density but a variable radius, Eq. (3) can be solved analytically and we obtain for the time $\left(t_{\text {photo }}\right)$ that it takes to completely photoevaporate the object

$t_{\text {photo }}=2.1 \times 10^{20} \phi_{49}^{-1 / 2}\left(2 \pi m_{\mathrm{H}} / 3\right)^{1 / 2} n_{3}^{1 / 2} d_{17} M^{1 / 2}\left[10^{6} \mathrm{yr}\right]$,

with the number density $n_{3}$ in $\left[10^{3} \mathrm{~cm}^{-3}\right]$ and the mass $M\left[M_{\odot}\right]$ from Table 1 , and the mass of hydrogen $m_{\mathrm{H}}$ [g]. In this simple approach, we neglected external compression which would increase $t_{\text {photo }}$ because it reduces the size (i.e., radius) of the object which in turn lowers the mass loss rate. We note also that the values for the distances are only approximations because there is no clearly defined center of the Cyg OB2 association, and there are line-of-sight effects. The time of exposure to UV radiation for the object is estimated by $t_{\text {expos }}=d_{\text {front }} / v_{\text {exp }} \mathrm{km} \mathrm{s}^{-1}$ with the expansion velocity of $v_{\text {exp }}$ of the $\mathrm{H}_{\text {II }}$ region. In this paper we use a value of $10 \mathrm{~km} \mathrm{~s}^{-1}$ for our calculations which is a typical value of expanding $\mathrm{H}_{\text {II }}$ regions (e.g., Williams et al. 2001).

This value is approximately in accord with that determined by comparing the relative velocity of the average radial stellar velocity of Cyg OB2 of $-28 \mathrm{~km} \mathrm{~s}^{-1}$ (Simbad data base) and the molecular cloud, that is -2 to $-11 \mathrm{~km} \mathrm{~s}^{-1}$ (Schneider et al. 2006). Another way to approximate $v_{\exp }$ is to use $v_{\mathrm{exp}}=R_{\mathrm{b}} / t_{\mathrm{OB} 2}$ with the extent of the bubble created by the $\mathrm{H}_{\text {II }}$ region $\left(R_{\mathrm{b}}\right)$, and the age of the Cyg OB2 association $\left(t_{\mathrm{OB} 2}\right)$. The average radius of the bubble is $\sim 40 \mathrm{pc}$, derived from, for example, MSX-images (Schneider et al. 2006) or the Canadian Galactic Plane Survey at $1.4 \mathrm{GHz}$ (Reipurth \& Schneider 2008). The cluster age is under discussion, with values ranging from 3-4 Myr (e.g., Comerón \& Pasquali 2012) and 5-7 Myr (e.g., Drew et al. 2008, Wright et al. 2015). We thus determine an upper limit of $\sim 17 \mathrm{~km} \mathrm{~s}^{-1}$ for $v_{\exp }$ and a lower limit of $\sim 7 \mathrm{~km} \mathrm{~s}^{-1}$. Considering the spread in values from the different methods, we take $10 \mathrm{~km} \mathrm{~s}^{-1}$ as a conservative value and estimate that the lifetime calculations are correct within a factor of 1.5-2.

The photoevaportation lifetimes can be compared to the freefall time for isothermal, gravitational collapse, as

$t_{\mathrm{ff}}=\sqrt{3 / 2 \pi G \rho}$,

in which $G$ is the gravitational constant and $\rho=n m_{\mathrm{H}}$ the average volume density of the structure studied.

The calculated lifetime values are listed in Table A.2. The most massive and extended objects - the pillars and globules survive photoevaporation the longest, up to $8 \times 10^{6} \mathrm{yr}$. There is one exception, pi7, which has $t_{\text {photo }}$ of only $5 \times 10^{5} \mathrm{yr}$. Condensations also have a relatively long photoevaporation lifetime $\left(\sim 2\right.$ to $\left.8 \times 10^{6} \mathrm{yr}\right)$. EGGs have shorter $t_{\text {photo }}$ of $1-2 \times 10^{6} \mathrm{yr}$. For proplyd-like objects the photoevaporation lifetime is in the order of only a few $10^{5} \mathrm{yr}$. This timescale is consistent with what was estimated for the circumstellar disks in Orion (Johnstone et al. 1998) though it is not clear if all objects are indeed photoevaporating disks. The majority of the proplyd-like objects $(\sim 70 \%)$ actually already have stars within them, as evidenced by optical (Guarcello et al. 2012) and near-IR (Wright et al. 2012) point sources detected within them and the spectroscopy of two of their central stars (Guarcello et al. 2014), see also Sect. 5. Interestingly, Gahm et al. (2007) found lifetimes of about $4 \times 10^{6} \mathrm{yr}$ for the globulettes in the Rosette Nebula, and IC1805, which have sizes smaller than $0.05 \mathrm{pc}$ and average densities of $3-100 \times 10^{3} \mathrm{~cm}^{-3}$. These longer photo evaporation times are likely due to much weaker FUV-field in these regions (both have far fewer O stars than Cyg OB2). Given our angular resolution, our census is not sensitive to these objects.

The ratio $t_{\text {photo }} / t_{\text {expos }}$ is an indicator of the evolutionary state of the structure. Pillars, globules, and condensations have an average ratio of $\sim 5, \sim 2$, and $\sim 3$, respectively, suggesting that they are mostly in an early state of their evolution. EGGs typically have a ratio of one, which may imply that they are already half way through of being dissociated. Finally, proplyd-like objects have very small ratios of $\sim 0.05$ and could be close to disappearing.

Taking objects' current densities (Table 1), we estimate freefall lifetimes ${ }^{3}$ typically of a few $10^{5}$ yr. Since $t_{\mathrm{ff}}$ is proportional to $1 / \sqrt{(} n)$, the densest objects - condensations - have the shortest collapse times, around $6 \times 10^{5} \mathrm{yr}$. The condensations, but also pillars, EGGs, and globules, are all potentially star-forming with $t_{\mathrm{ff}}<t_{\text {photo }}$ and $t_{\mathrm{ff}} \ll t_{\text {total }}$. Only proplyd-like objects have comparable lifetimes for photoevaporation and gravitational collapse. However, most of them, at least the original 10 from Wright et al. (2012), have central stars observed in the optical or nearIR bands. We suspect that the large envelopes surrounding these stars will be eroded and destroyed within a short period of time which might influence how much more mass these stars can accrete, thus determining their final stellar masses.

\subsection{An evolutionary sequence?}

Many detached structures (globules, proplyds, etc.) are observed in the Cygnus X region. The presence of such structures is also a signature found by Tremblin et al. (2012b), when the initial turbulence in the molecular cloud dominates over the compression of the ionized gas. Hence, the turbulent pressure is likely to be as important in Cygnus $\mathrm{X}$ as the ionized gas pressure. Interestingly, Gritschneder et al. (2009) found dense pillar heads in their simulations and less material at the bases, which is a morphology seen for all pillars in Cygnus X. This behavior is also observed in the controlled simulations of Tremblin et al. (2012a, Fig. 5) and indicates that the structures are relatively old (see below), with pillars stretched until their heads are completely separated from the rest of the cloud.

The controlled runs in Tremblin et al. (2012a) represent a scenario of the interaction between an ionization front and a medium of constant density with a modulated interface geometry or a flat interface with density enhancements. They show that pillars with a large width/height ratio $(\mathrm{w} / \mathrm{h})$ will take a very long time $(t)$ to grow. The narrowest pillar with an initial $w / h=0.5$ (see Fig. 6 of Tremblin et al. (2012a) reached a length of $1.5 \mathrm{pc}$ with $w / h=0.16$ in $5 \times 10^{5}$ yr. In Cygnus $\mathrm{X}$, the ratio $R=w / h$ (Table 1 ) is on average 0.22 , varying between 0.16 and 0.28 , and the length of the pillars is between $0.6 \mathrm{pc}$ and $3 \mathrm{pc}$ with an average of $1.35 \mathrm{pc}$. If these values are compared to the ones shown in Tremblin et al. (2012a), see also Kinnear et al. (2014, 2015), the

\footnotetext{
3 We note that $t_{\mathrm{ff}}$ is a lower limit, the collapse time increases if the structure has support by turbulence, pressure, rotation, or magnetic
} fields. 
pillars observed in Cygnus $\mathrm{X}$ are likely to be quite well evolved. Only the curve with the smallest $\mathrm{w} / \mathrm{h}$ ratio with large pillar sizes $(>1 \mathrm{pc})$ is comparable to our observed values and hence indicates an evolutionary state of $t>5 \times 10^{5} \mathrm{yr}$. This lower limit of $\mathrm{t}$ is consistent with the exposure time $t_{\text {expos }}$ we calculated in Sect. 7.1 which ranges between $2 \times 10^{5} \mathrm{yr}$ and $4 \times 10^{6} \mathrm{yr}$.

Putting together the physical properties determined from the Herschel observations (Sect. 5.2), the lifetime calculations (Sect. 6.1), and the results from comparison with simulations (see above), we propose a tentative evolutionary scheme for the observed features.

Pillars and globules are the most massive objects we see and they correspond in their physical properties (average size, density, and mass of $\sim 1 \mathrm{pc}, 5-10 \times 10^{3} \mathrm{~cm}^{-3}$, and $\sim 500 M_{\odot}$, respectively) to typical molecular cloud clumps. Condensations, and to a lesser extent EGGs, are likely molecular cloud dense cores with typical size and density of $0.1 \mathrm{pc}$ and $2-6 \times 10^{4} \mathrm{~cm}^{-3}$, respectively. In this scheme, proplyd-like objects correspond to less dense $\left(10^{3} \mathrm{~cm}^{-3}\right)$ cores. The main difference between these objects is that clumps and cores form as a part of a molecular cloud and are embedded in the clouds (e.g., cores within a filament), whereas globules, EGGs, condensations, and proplyd-like objects are isolated features. The resemblence of their physical properties, however, may suggest a filamentary origin as a possible scenario (see Introduction).

The remaining molecular envelopes of proplyd-like objects are less massive than those in the EGGs, probably because some of the mass has gone into the forming stars. Pillars form a special category because they are still attached to the cloud. This fact points towards a scenario in which pillars can be the eroded leftovers of a pre-existing clump or filament (Dale et al. 2015). Tremblin et al. (2012a,b) show that pillars can form either in a density- or surface-modulated region.

We find that pillars have the longest timescales for photoevaporation, mainly because they are massive and large, and have the smallest times of UV-exposure. We speculate that a pillar evolves into a globule and then on into a condensation. The major difference between globule and condensation is that the latter has no head-tail structure and is a factor of four denser and smaller. Larger globules might form multiple stars (see the example of globule g1 where several B-stars were found), possibly leading to multiple proplyd-like objects grouped together. This would also explain why the proplyd-like objects are grouped together a few at a time in little clumps. A condensation may be an evolved globule in which most of the lower density gas has photoevaporated away, leaving only a dense $\left(5.5 \times 10^{4} \mathrm{~cm}^{-3}\right)$ and cold $(T \sim 15 \mathrm{~K})$ core. We further speculate that EGGs and proplyd-like objects could be also leftovers of initially larger globules. As their name already indicates, EGGs, that is evaporating gaseous globules, have globular shapes. They are, however, less dense $\left(2 \times 10^{4} \mathrm{~cm}^{-3}\right)$ and warm $(T \sim 17 \mathrm{~K})$ and thus prone to disappear fast, in contrast to condensations that are potential sites of star/cluster formation. Proplyd-like objects may share the same fate as EGGs but they are on average more massive and extended and so likely to survive the photoevaporating impact of Cyg OB2 for longer. Given that some of them show signatures of star-formation activity, they could be the leftovers of condensations. If proplyd-like objects follow EGGs and if the latter have time to form stars, then it is not unreasonable to think that the former may have formed stars but still be surrounded by a portion of an envelope that is still finishing evaporating.

\section{Summary}

We used Herschel FIR imaging observations of the Cyg OB2 region, performed within the HOBYS keyprogram, to detect and characterize features that are formed in the interface region between $\mathrm{H}_{\text {II }}$ region and molecular cloud. Using a $400 \mathrm{MJy} / \mathrm{sr}$ flux threshold in the $70 \mu \mathrm{m}$ map, we define pillars, globules, evaporating gaseous globules (EGGs), proplyd-like objects, and condensations. From SED fits to the 160-500 $\mu \mathrm{m}$ Herschel wavelengths, we determine column density and temperature maps, and derive masses, volume densities, and surface densities for these structures. From the $70 \mu \mathrm{m}$ and $160 \mu \mathrm{m}$ flux maps, we estimate an average FUV-field of typically a few hundred Habing on the photon dominated surfaces. We find that the initial morphological classification indeed corresponds to distinct objects with different physical properties.

- Pillars are the largest structures (equivalent mean average radius $\langle r\rangle \sim 0.6 \mathrm{pc}$ ). They have an average density of $\langle n\rangle \sim$ $5 \times 10^{3} \mathrm{~cm}^{-3}$, and an average temperature $\langle T\rangle$ of $18 \mathrm{~K}$. They often show temperature gradients along their longer axis. Their masses range between $50 M_{\odot}$ and $1680 M_{\odot}$, with an average mass $\langle M\rangle \sim 500 M_{\odot}$.

- Globules are also large $(\langle r\rangle \sim 0.6 \mathrm{pc})$ but they have a more defined head-tail structure with a denser "head" than pillars with $\langle n\rangle \sim 1.2 \times 10^{4} \mathrm{~cm}^{-3}$. Their average mass and temperature are $\sim 500 M_{\odot}$ and $\sim 18 \mathrm{~K}$, respectively, similar to pillars.

- EGGs and proplyd-like objects are smaller $(r \sim 0.1$ and $0.2 \mathrm{pc})$ and less massive ( $\sim 10 M_{\odot}$ and $\sim 30 M_{\odot}$, respectively), but they have a high average density of $2.2 \times 10^{4} \mathrm{~cm}^{-3}$ and $1.5 \times 10^{4} \mathrm{~cm}^{-3}$, respectively. They both have an average temperature of $17 \mathrm{~K}$.

- Condensations are small ( $\sim 0.1 \mathrm{pc})$, have an average mass of $35 M_{\odot}$, and are the densest structures we found in our sample with $\langle n\rangle \sim 5.5 \times 10^{4} \mathrm{~cm}^{-3}$.

In summary, pillars and globules are irradiated structures which correspond to a subset of what is described as "clumps" in molecular clouds while irradiated condensations correspond to a subset of massive dense molecular cloud "cores". All pillars, globules and proplyd-like objects show a clear orientation toward the center of the Cyg OB2 association. They are thus directly influenced by the radiation of the stars and we used a census of them to estimate the lifetimes of all observed features using a model for photoevaporating dense clumps (Johnstone et al. 1998). Pillars and globules have the longest estimated photoevaporation lifetimes, a few million years, while all other features most likely survive less than a million years. These lifetimes are consistent with what was found in simulations of turbulent, UV-illuminated clouds (Tremblin et al. 2012a,b). We propose a tentative evolutionary scheme in which pillars can evolve into globules, which in turn then evolve into EGGs, condensations and proplyd-like objects.

Acknowledgements. SPIRE has been developed by a consortium of institutes led by Cardiff University (UK) and including Univ. Lethbridge (Canada); NAOC (China); CEA, LAM (France); IFSI, Univ. Padua (Italy); IAC (Spain); Stockholm Observatory (Sweden); Imperial College London, RAL, UCL-MSSL, UKATC, Univ. Sussex (UK); and Caltech, JPL, NHSC, Univ. Colorado (USA). This development has been supported by national funding agencies: CSA (Canada); NAOC (China); CEA, CNES, CNRS (France); ASI (Italy); MCINN (Spain); SNSB (Sweden); STFC (UK); and NASA (USA). PACS has been developed by a consortium of institutes led by MPE (Germany) and including UVIE (Austria); KU Leuven, CSL, IMEC (Belgium); CEA, LAM (France); MPIA (Germany); INAF-IFSI/OAA/OAP/OAT, LENS, SISSA (Italy); IAC (Spain). This development has been supported by the funding agencies BMVIT (Austria), ESA-PRODEX (Belgium), CEA/CNES (France), DLR (Germany), ASI/INAF 
(Italy), and CICYT/MCYT (Spain). Part of this work was supported by the ANR 11-BS56-010 project "STARFICH" and the ERC advanced Grant no. 291294 "ORISTARS". N.S. acknowledges support from the DFG-priority program 1573 (ISM-SPP), through project number Os $177 / 2-1$ and 177/2-2. N.J.W. acknowledges an RAS Research Fellowship. We thank the referee, G. Gahm for his competent comments that improved the clarity of the paper.

\section{References}

André, Ph., Men'shchikov, A., Bontemps, S., et al. 2010, A\&A, 518, L102 André, Ph., Di Francesco, J., Ward-Thompson, D., et al. 2014, Protostars and Planets VI, eds. H. Beuther, R. S. Klessen, C. P. Dullemond, \& Th. Henning (University of Arizon Press), 27

Anderson, L. D., Zavagno, A., Deharveng, L., et al. 2012, A\&A, 542, A10 Bertoldi, F., 1989, ApJ, 346, 735

Bernard, J.-P., Paradis, D., Marshall, D. J., et al. 2010, A\&A, 518, L88

Comerón, F., \& Pasquali, A. 2012, A\&A, 543, A101

Comerón, F., \& Torra, J. 1999, A\&A, 349, 605

Comerón, F., Pasquali, A., Figueras, F., \& Torra, J. 2008, A\&A, 486, 453

Dale, J. E., Ngoumou, J., Ercolano, B., \& Bonnell, I. A. 2014, MNRAS, 442, 694

Dale, J. E., Haworth, T. J., \& Bressert, E. 2015, MNRAS, 450, 1199

Deharveng, L., Zavagno, A., Anderson, L. D., et al. 2012, A\&A, 546, A74

Didelon, P., Motte, F., Tremblin, P., et al. 2015, A\&A, 584, A4

Drew, J. E., Greimel, R., Irwin, M. J., \& Sale, S. E. 2008, MNRAS, 386, 1761

Gahm, G. F., Grenman, T., Frederiksson, S., \& Kristen, H. 2007, ApJ, 133, 1795

Griffin, M., Abergel, A., Abreau, A., et al. 2010, A\&A, 518, L3

Gritschneder, M., Naab, T., Walch, S., et al. 2009, ApJ, 694, L26

Gritschneder, M., Burkert, A., Naab, T., \& Walch, S. 2010, ApJ, 723, 971

Guarcello, M. G., Wright, N. J., Drake, J. J., et al. 2012, ApJS, 202, 19

Guarcello, M. G., Drake, J. J., Wright, N. J., et al. 2013, ApJ, 773, 135

Guarcello, M. G., Drake, J. J., Wright, N. J., et al. 2014, ApJ, 793, 56

Habing, H.J. 1968, Bull. Astron. Inst. Netherlands, 19, 421

Hanson, M. M. 2003, ApJ, 597, 957

Hennemann, M., Motte, F., Schneider, N., et al. 2012, A\&A, 543, L3

Herbig, G. 1974, PASP, 86, 604

Hester, J. J., Scowen, P. A., \& Sankrit, R. 1996, AJ, 111, 2349

Hill, T., Motte F., Didelon P., et al. 2011, A\&A, 533, A4

Hill, T., Motte F., Didelon P., et al. 2012, A\&A, 542, A12

Johnstone, D., Hollenbach, D., \& Bally, J. 1998, ApJ, 499, 758

Kinnear, T. M., Miao, J., White, G. J., \& Goodwin, S. 2014, MNRAS, 444, 122

Kinnear, T.M., Miao, J., White, G.J., et al. 2015, MNRAS, 450, 1017

Könyves, V., André, Ph., Men'shchikov, A., et al. 2015, A\&A, 584, A33

Kramer, C., Cubick, M., Röllig, M., et al. 2008, A\&A, 477, 547
Laques, P., \& Vidal, J. L. 1979, A\&A, 73, 97

Lefloch, B., \& Lazareff, B. 1994, A\&A, 289, 559

Lo, N., Cunningham, M. R., Jones, P. A., et al. 2009, MNRAS, 395, 1021

Martins, F., Schaerer, D., \& Hillier, D. J. 2005, A\&A, 436, 1049

McCaughrean, M. J., \& Andersen, M. 2002, A\&A, 390, L27

Miao, J., White, G. J., Nelson, R. P., et al. 2006, MNRAS, 369, 143

Miao, J., White, G. J., Thompson, M. A., \& Nelson, R. P. 2009, ApJ, 692, 382

Minier, V., Tremblin, P., Hill, T., et al. 2013, A\&A, 550, A50

Motte, F., Bontemps, S., Schilke, P., et al. 2007, A\&A, 476, 1243

Motte, F., Zavagno, A., Bontemps, S., et al. 2010, A\&A, 518, L77

Odenwald, S. F., Campbell, M. F., Shivanandan, K., et al. 1990, ApJ, 99, 288

O’Dell, C. R., Wen, Z., \& Hu, X. 1993, ApJ, 410, 696

Piddington, J. H., \& Minnett, H. C. 1952, Austr. J. Sci. Res. A, 5, 17

Pilbratt, G., Riedinger, J., Passvogel, T., et al. 2010, A\&A 518, L1

Poglitsch, A., Waelkens, C., Geis, N., et al. 2010, A\&A, 518, L2

Reipurth, B., \& Schneider, N. 2008, Handbook of star-forming regions, ASP, 37

Roccatagliata, V., Preibisch, T., Ratzka, T., \& Gaczkowski, B. 2013, A\&A, 554, A16

Roussel, H. 2013, PASP, 125, 1126

Roy, A., Martin, P., \& Polychroni, D., 2013, ApJ, 763, 55

Roy, A., André, Ph., Palmeirim, P., et al. 2014, A\&A, 562, A10

Rygl, K., Brunthaler, A., Sanna, A., et al. 2012, A\&A 539, A12

Schneider, N., Stutzki, J., Winnewisser, G., \& Block, D. 1998, A\&A, 335, 1049

Schneider, N., Bontemps, S., Simon, R., et al. 2006, A\&A, 458, 855

Schneider, N., Motte, F., Bontemps, S., et al. 2010, A\&A, 518, L83

Schneider, N., Simon, R., Bontemps, S., et al. 2011, A\&A, 529, A1

Schneider, N., Güsten R., Tremblin P., et al. 2012a, A\&A, 542, L18

Schneider, N., Csengeri, T., Hennemann, M., et al. 2012b, A\&A, 540, L11

Schneps, M. H, Ho, P. T. P., \& Barrett, A. H, 1980, ApJ, 240, 84

Smith, N., Bally, J., \& Morse, J. A. 2003, ApJ, 587, L105

Sternberg, A., Hoffmann, T. L., \& Pauldrach, A. W. A. 2003, ApJ, 599, 1333

Sugitani, K., Tamura, M., Nakajima, Y., et al. 2002, ApJ, 565, L28

Tremblin, P., Audit, E., Minier, V., \& Schneider, N. 2012a, A\&A, 538, A12

Tremblin, P., Audit, E., Minier, V., Schmidt, W., \& Schneider, N. 2012b, A\&A, 546, A11

Tremblin, P., Minier, V., \& Schneider, N. 2013, A\&A, 560, A11

Tremblin, P., Schneider, N., \& Minier, V. 2014, A\&A, 564, A13

White, G., Lefloch, B., Fridlund, C. V. M., et al. 1997, A\&A, 323, 931

White, G., Nelson, R. P., Holland, W. S., et al. 1999, A\&A, 342, 233

White, G., Abergel, A., Spencer, L., et al. 2010, A\&A, 518, L114

Williams, R. J. R., Ward-Thompson, D., Whitworth, A. P. 2001, MNRAS, 327, 788

Wright, N., Drake, J., Drew, J. E., et al. 2012, ApJ, 746, L21

Wright, N., Drew, J. E., Mohr-Smith, M., 2015, MNRAS, 449, 741

Zavagno, A., Anderson, L., Russeil, D., et al. 2010, A\&A, 518, L101 


\section{Appendix A: Herschel images at 70 to $500 \mu \mathrm{m}$ of all regions}

Table A.1. Physical properties of pillars, globules, EGGS, proplyd-like, and condensations.

\begin{tabular}{|c|c|c|c|c|c|c|c|c|c|c|}
\hline Source & $\begin{array}{c}\left\langle N\left(\mathrm{H}_{2}\right)\right\rangle \\
{\left[10^{21} \mathrm{~cm}^{-2}\right]} \\
(1)\end{array}$ & $\begin{array}{c}M \\
{\left[M_{\odot}\right]} \\
(2) \\
\end{array}$ & $\begin{array}{c}\left\langle n\left(\mathrm{H}_{2}\right)\right\rangle \\
{\left[10^{3} \mathrm{~cm}^{-3}\right]} \\
(3)\end{array}$ & $\begin{array}{l}\langle T\rangle \\
{[\mathrm{K}]} \\
(4) \\
\end{array}$ & $\begin{array}{l}T_{\min } \\
{[\mathrm{K}]} \\
(5) \\
\end{array}$ & $\begin{array}{c}T_{\max } \\
{[\mathrm{K}]} \\
(6) \\
\end{array}$ & $\begin{array}{c}r \\
{[\mathrm{pc}]} \\
(7) \\
\end{array}$ & $\begin{array}{c}l \times w \\
{[\mathrm{pc} \times \mathrm{pc}]} \\
(8)\end{array}$ & $\begin{array}{c}\Sigma \\
{\left[M_{\odot} / \mathrm{pc}^{2}\right]} \\
(9)\end{array}$ & $\begin{array}{c}\text { Flux }\rangle \\
{[\text { Go] }} \\
(10) \\
\end{array}$ \\
\hline \multicolumn{11}{|c|}{ Pillars } \\
\hline 1 & 21.0 & 1680 & 2.9 & 17.3 & 14.8 & 18.7 & 1.17 & $2.86 \times 0.46$ & 390 & 198 \\
\hline 2 & 11.5 & 282 & 3.1 & 17.7 & 17.4 & 18.2 & 0.65 & $1.22 \times 0.31$ & 213 & 147 \\
\hline 3 & 16.1 & 83 & 8.8 & 17.1 & 16.7 & 17.3 & 0.30 & $0.61 \times 0.17$ & 298 & 177 \\
\hline 4 & 11.5 & 50 & 6.9 & 17.3 & 16.8 & 17.4 & 0.27 & $0.63 \times 0.13$ & 213 & 122 \\
\hline 5 & 17.2 & 156 & 7.1 & 17.1 & 16.2 & 17.4 & 0.39 & $0.87 \times 0.18$ & 319 & 175 \\
\hline 6 & 21.0 & 1403 & 3.2 & 18.0 & 17.3 & 20.2 & 1.07 & $2.38 \times 0.43$ & 390 & 295 \\
\hline 7 & 7.9 & 86 & 3.0 & 19.1 & 18.1 & 20.1 & 0.43 & $0.88 \times 0.24$ & 147 & 191 \\
\hline Mean & $15.2 \pm 1.9$ & $534 \pm 263$ & $5.0 \pm 0.9$ & $17.7 \pm 0.3$ & $16.8 \pm 0.4$ & $18.5 \pm 0.5$ & $0.61 \pm 0.14$ & & $281 \pm 35$ & $186 \pm 21$ \\
\hline \multicolumn{11}{|c|}{ Globules } \\
\hline 1 & 14.2 & 238 & 4.3 & 19.7 & 17.2 & 26.3 & 0.54 & & 263 & 549 \\
\hline 2 & 22.1 & 108 & 23.8 & 16.1 & 15.7 & 16.5 & 0.29 & & 410 & 156 \\
\hline 3 & 30.6 & 180 & 15.8 & 17.5 & 15.6 & 20.2 & 0.31 & & 568 & 294 \\
\hline 4 & 15.0 & 1356 & 2.0 & 19.7 & 18.6 & 23.7 & 1.24 & & 279 & 428 \\
\hline Mean & $20.5 \pm 3.8$ & $470 \pm 296$ & $12 \pm 5$ & $18.3 \pm 0.9$ & $16.8 \pm 0.7$ & $21.7 \pm 2.1$ & $0.60 \pm 0.22$ & & $380 \pm 71$ & $357 \pm 85$ \\
\hline \multicolumn{11}{|c|}{ EGGs } \\
\hline 1 & 12.9 & 10.8 & 18.6 & 17.1 & & & 0.11 & & 239 & 137 \\
\hline 2 & 11.7 & 5.9 & 22.8 & 17.0 & & & 0.08 & & 217 & 113 \\
\hline 3 & 13.5 & 38.6 & 10.1 & 17.3 & & & 0.22 & & 251 & 153 \\
\hline 4 & 13.5 & 4.5 & 34.4 & 16.9 & & & 0.06 & & 251 & 137 \\
\hline 5 & 12.8 & 6.4 & 25.0 & 17.1 & & & 0.08 & & 238 & 127 \\
\hline Mean & $12.9 \pm 0.3$ & $13 \pm 6$ & $22 \pm 4$ & $17.1 \pm 0.1$ & & & $0.11 \pm 0.03$ & & $239 \pm 6$ & $133 \pm 7$ \\
\hline \multicolumn{11}{|c|}{ Proplyd-like } \\
\hline 1 & 10.9 & 11.0 & 13.5 & 17.1 & & & 0.12 & $0.12 \times 0.08$ & 202 & 160 \\
\hline 2 & 10.5 & 5.3 & 20.4 & 17.8 & & & 0.08 & $0.31 \times 0.11$ & 194 & 211 \\
\hline 3 & 16.2 & 48.8 & 11.7 & 17.8 & & & 0.22 & $0.55 \times 0.29$ & 300 & 394 \\
\hline 4 & 13.1 & 24.2 & 12.3 & 17.6 & & & 0.17 & $0.49 \times 0.16$ & 243 & 315 \\
\hline 5 & 17.4 & 69.9 & 10.9 & 17.2 & & & 0.26 & $0.36 \times 0.20$ & 322 & 340 \\
\hline $5 a$ & 13.8 & 37.0 & 10.6 & 17.2 & & & 0.21 & & 256 & 192 \\
\hline $5 b$ & 12.7 & 23.5 & 12.0 & 17.1 & & & 0.17 & & 236 & 157 \\
\hline $5 c$ & 12.7 & 17.0 & 14.1 & 17.0 & & & 0.15 & & 235 & 171 \\
\hline $5 d$ & 12.3 & 12.4 & 16.0 & 17.2 & & & 0.12 & & 229 & 179 \\
\hline 6 & 21.1 & 84.9 & 13.2 & 16.9 & & & 0.26 & $0.37 \times 0.13$ & 392 & 296 \\
\hline 7 & 12.8 & 42.8 & 8.8 & 18.3 & & & 0.24 & $0.37 \times 0.11$ & 237 & 349 \\
\hline 8 & 13.7 & 50.5 & 9.0 & 17.2 & & & 0.25 & $0.21 \times 0.07$ & 254 & 314 \\
\hline 9 & 10.5 & 3.5 & 26.8 & 17.4 & & & 0.06 & $0.09 \times 0.06$ & 195 & 138 \\
\hline 10 & 14.6 & 26.9 & 13.7 & 17.7 & & & 0.17 & $0.40 \times 0.13$ & 270 & 255 \\
\hline 11 & 26.0 & 34.9 & 28.9 & 17.7 & & & 0.15 & & 483 & 394 \\
\hline 12 & 10.2 & 8.5 & 14.7 & 17.3 & & & 0.11 & & 189 & 128 \\
\hline 13 & 11.8 & 11.9 & 15.4 & 17.4 & & & 0.12 & & 219 & 144 \\
\hline Mean & $14.1 \pm 1.0$ & $31 \pm 6$ & $15 \pm 1$ & $17.4 \pm 0.09$ & & & $0.17 \pm 0.02$ & & $262 \pm 19$ & $243 \pm 23$ \\
\hline \multicolumn{11}{|c|}{ Condensations } \\
\hline 1 & 32.6 & 21.9 & 53.5 & 14.4 & & & 0.10 & & 605 & 110 \\
\hline 2 & 26.3 & 8.8 & 67.0 & 15.1 & & & 0.06 & & 488 & 123 \\
\hline 3 & 29.8 & 49.8 & 29.4 & 15.3 & & & 0.16 & & 552 & 122 \\
\hline 4 & 32.4 & 21.7 & 53.1 & 15.5 & & & 0.10 & & 601 & 168 \\
\hline 5 & 42.4 & 21.3 & 82.7 & 15.1 & & & 0.08 & & 787 & 156 \\
\hline 6 & 59.3 & 69.5 & 70.9 & 15.1 & & & 0.14 & & 1100 & 272 \\
\hline 7 & 25.0 & 30.0 & 30.0 & 15.3 & & & 0.14 & & 465 & 131 \\
\hline Mean & $35.4 \pm 4.5$ & $35 \pm 5$ & $55 \pm 8$ & $15.1 \pm 0.1$ & & & $0.11 \pm 0.01$ & & $657 \pm 84$ & $155 \pm 21$ \\
\hline
\end{tabular}

Notes. The last line of each section gives the average (mean) values for each class of sources in bold. (1) Average column density derived within the $70 \mu \mathrm{m}$ contour level. (2) Mass derived from column density map within the contours of the $70 \mu \mathrm{m}$ data. (3) Average density from the mass $M$, assuming a spherical shape with an equivalent radius $r$. (4) Average temperature (average across the area covered by the $70 \mu \mathrm{m}$ contour). (5) And (6) Minimum and maximum temperature. (7) Equivalent radius $(r=\sqrt{\text { area } / \pi})$, deconvolved with the beam $\left(6^{\prime \prime}\right.$ for $70 \mu \mathrm{m}$ that corresponds to $0.04 \mathrm{pc}$ for a distance of $1.4 \mathrm{kpc}$ ). (8) Length and width for pillars (this study) and proplyd-like (sizes from Wright et al. 2012). (9) Surface density. (10) Average UV-flux in units of Habing field. 
Table A.2. Photoevaporation-, total-, and free-fall lifetimes of observed objects.

\begin{tabular}{|c|c|c|c|c|c|}
\hline Source & $\begin{array}{c}d_{\mathrm{OB}} \\
{[\mathrm{pc}]} \\
(1)\end{array}$ & $\begin{array}{c}t_{\text {photo }} \\
{\left[10^{6} \mathrm{yr}\right]} \\
(2)\end{array}$ & $\begin{array}{c}t_{\text {expos }} \\
{\left[10^{6} \mathrm{yr}\right]} \\
(3)\end{array}$ & $\begin{array}{c}t_{\text {total }} \\
{\left[10^{6} \mathrm{yr}\right]} \\
(4)\end{array}$ & $\begin{array}{c}t_{\mathrm{ff}} \\
{\left[10^{6} \mathrm{yr}\right]} \\
(5)\end{array}$ \\
\hline \multicolumn{6}{|c|}{ Pillars $\left\langle n\left(\mathrm{H}_{2}\right)\right\rangle=5 \times 10^{3} \mathrm{~cm}^{-3},\langle r\rangle=0.61 \mathrm{pc}$} \\
\hline 1 & 43.4 & 8.38 & 0.92 & 9.30 & 0.14 \\
\hline 2 & 46.2 & 3.77 & 0.64 & 4.41 & 0.15 \\
\hline 3 & 50.4 & 3.76 & 0.23 & 3.99 & 0.25 \\
\hline 4 & 44.8 & 2.30 & 0.78 & 3.08 & 0.22 \\
\hline 5 & 30.8 & 2.84 & 2.15 & 4.99 & 0.23 \\
\hline 6 & 19.6 & 3.63 & 3.24 & 6.87 & 0.15 \\
\hline 7 & 10.5 & 0.47 & 4.13 & 4.60 & 0.15 \\
\hline \multicolumn{6}{|c|}{ Globules $\left\langle n\left(\mathrm{H}_{2}\right)\right\rangle=12 \times 10^{3} \mathrm{~cm}^{-3},\langle r\rangle=0.60 \mathrm{pc}$} \\
\hline 1 & 26.6 & 2.35 & 2.56 & 4.91 & 0.16 \\
\hline 2 & 36.4 & 5.10 & 1.60 & 6.70 & 0.41 \\
\hline 3 & 42.0 & 6.19 & 1.05 & 7.24 & 0.34 \\
\hline 4 & 11.5 & 1.66 & 4.03 & 5.69 & 0.12 \\
\hline \multicolumn{6}{|c|}{ Condensations $\left\langle n\left(\mathrm{H}_{2}\right)\right\rangle=55 \times 10^{3} \mathrm{~cm}^{-3},\langle r\rangle=0.11 \mathrm{pc}$} \\
\hline 1 & 37.8 & 3.58 & 1.46 & 5.04 & 0.62 \\
\hline 2 & 37.8 & 2.54 & 1.46 & 4.00 & 0.69 \\
\hline 3 & 37.8 & 4.00 & 1.46 & 5.46 & 0.46 \\
\hline 4 & 32.2 & 3.02 & 2.01 & 5.03 & 0.62 \\
\hline 5 & 33.8 & 3.92 & 1.86 & 5.78 & 0.77 \\
\hline 6 & 39.7 & 7.71 & 1.28 & 8.99 & 0.71 \\
\hline 7 & 29.4 & 2.44 & 2.29 & 4.73 & 0.46 \\
\hline \multicolumn{6}{|c|}{ EGGs $\left\langle n\left(\mathrm{H}_{2}\right)\right\rangle=22 \times 10^{3} \mathrm{~cm}^{-3},\langle r\rangle=0.11 \mathrm{pc}$} \\
\hline 1 & 37.8 & 1.48 & 1.47 & 2.95 & 0.37 \\
\hline 2 & 38.1 & 1.22 & 1.44 & 2.66 & 0.40 \\
\hline 3 & 38.6 & 2.04 & 1.39 & 3.43 & 0.27 \\
\hline 4 & 37.1 & 1.28 & 1.54 & 2.82 & 0.50 \\
\hline 5 & 37.8 & 1.32 & 1.47 & 2.79 & 0.42 \\
\hline \multicolumn{6}{|c|}{ Proplyd-like $\left\langle n\left(\mathrm{H}_{2}\right)\right\rangle=15 \times 10^{3} \mathrm{~cm}^{-3},\langle r\rangle=0.17 \mathrm{pc}$} \\
\hline 1 & 14.0 & 0.47 & 3.79 & $4.26^{1}$ & 0.31 \\
\hline 2 & 13.0 & 0.37 & 3.88 & 4.25 & 0.38 \\
\hline 3 & 11.5 & 0.76 & 4.03 & 4.79 & 0.29 \\
\hline 4 & 12.0 & 0.57 & 3.98 & 4.55 & 0.30 \\
\hline 5 & 13.0 & 0.99 & 3.88 & 4.87 & 0.28 \\
\hline $5 a$ & 13.2 & 0.72 & 3.86 & 4.58 & 0.28 \\
\hline $5 b$ & 13.4 & 0.62 & 3.85 & 4.47 & 0.29 \\
\hline $5 c$ & 13.0 & 0.56 & 3.88 & 4.44 & 0.32 \\
\hline $5 d$ & 12.7 & 0.49 & 3.91 & 4.40 & 0.34 \\
\hline 6 & 5.9 & 0.55 & 4.58 & 5.13 & 0.31 \\
\hline 7 & 6.4 & 0.34 & 4.52 & 4.86 & 0.25 \\
\hline 8 & 6.1 & 0.36 & 4.56 & 4.92 & 0.25 \\
\hline 9 & 6.9 & 0.18 & 4.48 & 4.66 & 0.44 \\
\hline 10 & 9.3 & 0.49 & 4.24 & 4.73 & 0.31 \\
\hline 11 & 37.8 & 1.47 & 1.46 & 2.93 & 0.45 \\
\hline 12 & 37.8 & 0.61 & 1.46 & 2.07 & 0.32 \\
\hline 13 & 32.2 & 0.11 & 2.01 & 2.12 & 0.33 \\
\hline
\end{tabular}

Notes. (1) Distance to the center of Cyg OB2. (2) Lifetime of object until complete destruction by photoevaporation. (3) Time the object has been exposed to UV radiation of the H II region. (4) Total lifetime $\left(t_{\text {photo }}+t_{\text {expos }}\right)$. (5) Free-fall time (Eq. (6)). 
N. Schneider et al.: Far-IR imaging of Cyg OB2
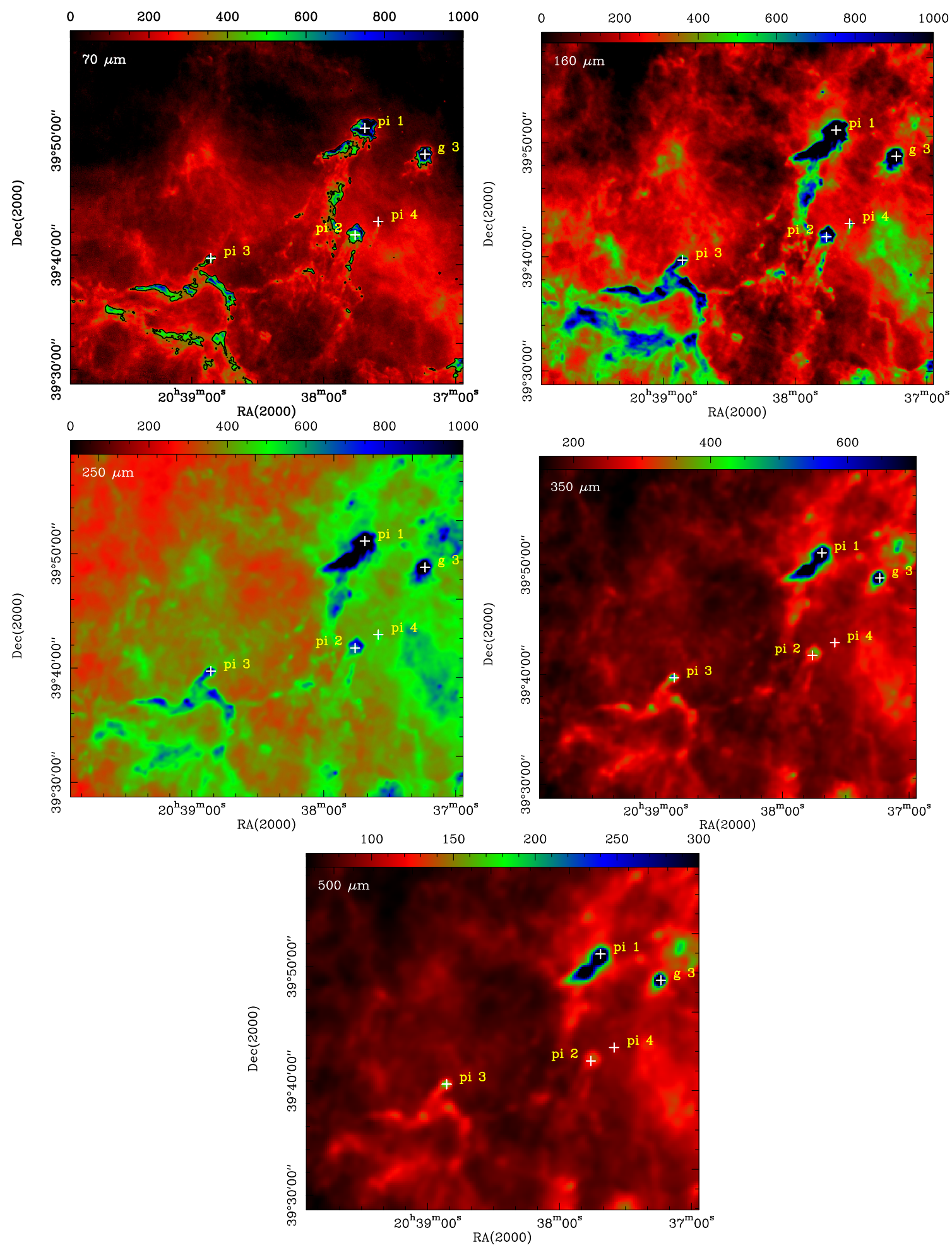

Fig. A.1. PACS (70 and $160 \mu \mathrm{m})$ and SPIRE $(250,350,500 \mu \mathrm{m})$ images of region 1-1. All maps are in units of MJy/sr and the features classified as pillars ("pi"), globules ("g"), condensations ("c") and EGGS ("e") are indicated by yellow crosses in the plots. The black contour in the $70 \mu \mathrm{m}$ map outlines the $400 \mathrm{MJy} / \mathrm{sr}$ level that was used to approximately define the shapes of the various features. 
A\&A 591, A40 (2016)
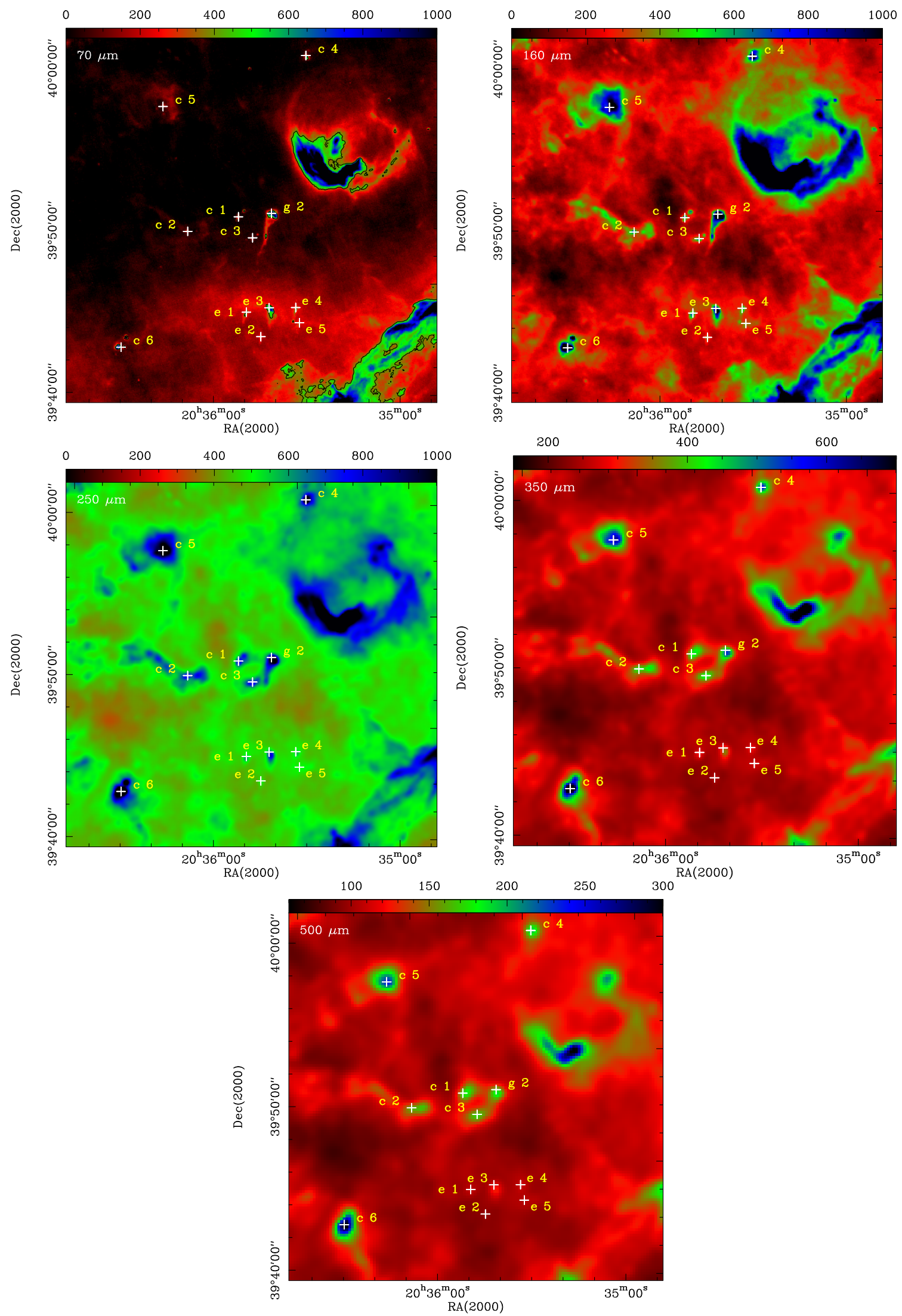

Fig. A.2. PACS (70 and $160 \mu \mathrm{m})$ and $\operatorname{SPIRE}(250,350,500 \mu \mathrm{m})$ images of region 1-2. 
N. Schneider et al.: Far-IR imaging of Cyg OB2
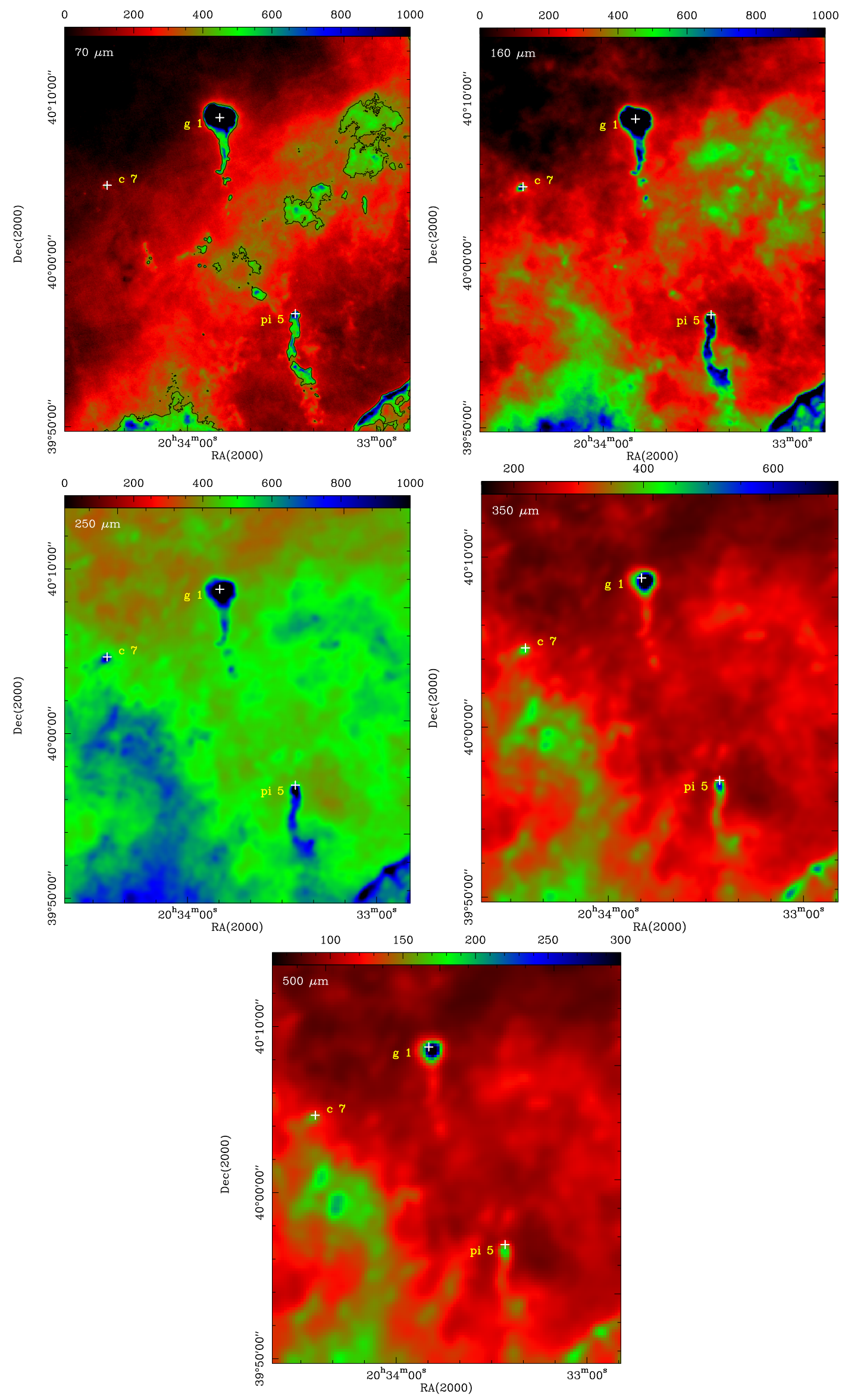

Fig. A.3. PACS (70 and $160 \mu \mathrm{m})$ and $\operatorname{SPIRE}(250,350,500 \mu \mathrm{m})$ images of region 1-3. 
A\&A 591, A40 (2016)
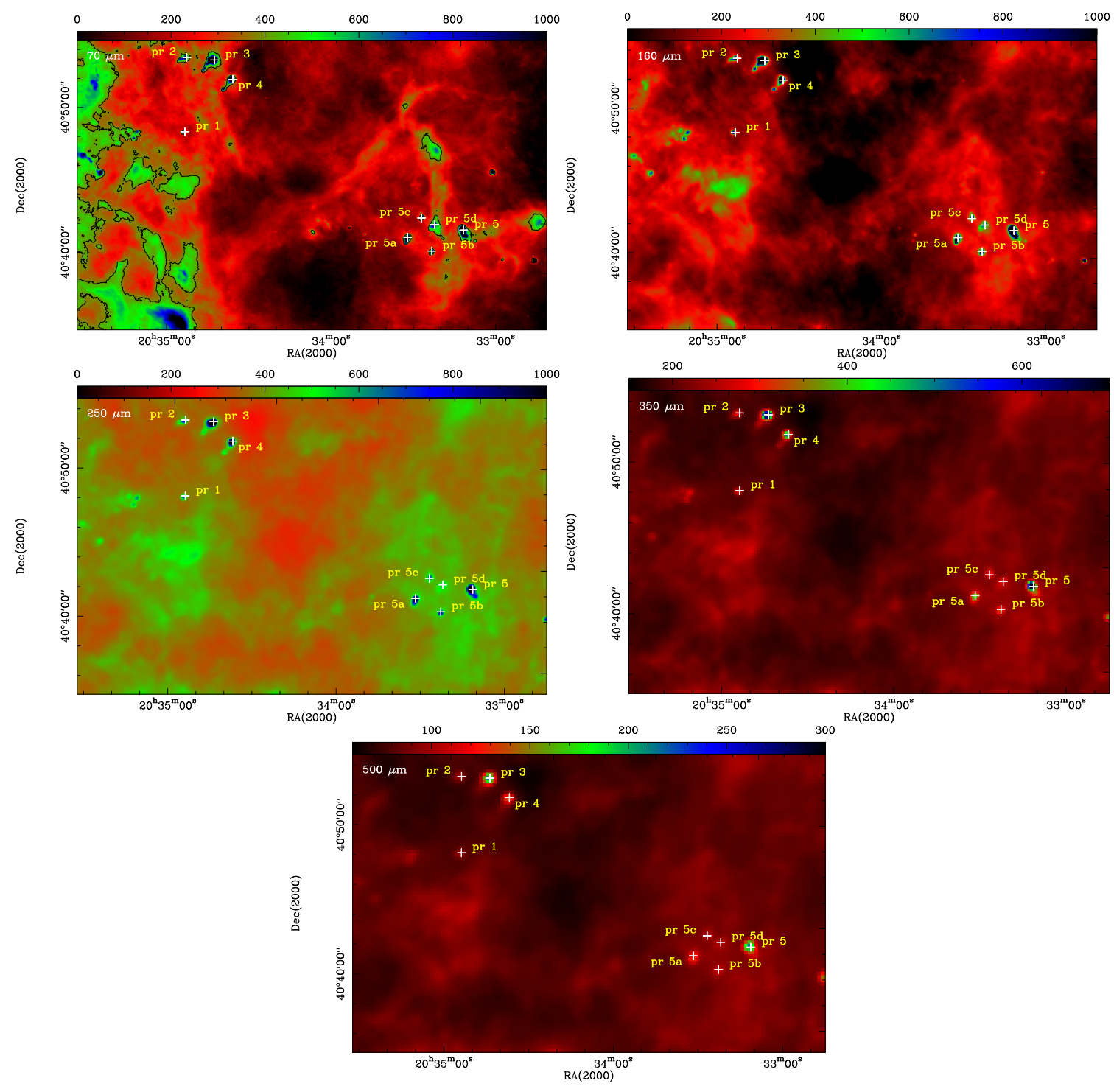

Fig. A.4. PACS (70 and $160 \mu \mathrm{m})$ and $\operatorname{SPIRE}(250,350,500 \mu \mathrm{m})$ images of region 2-1. 
N. Schneider et al.: Far-IR imaging of Cyg OB2

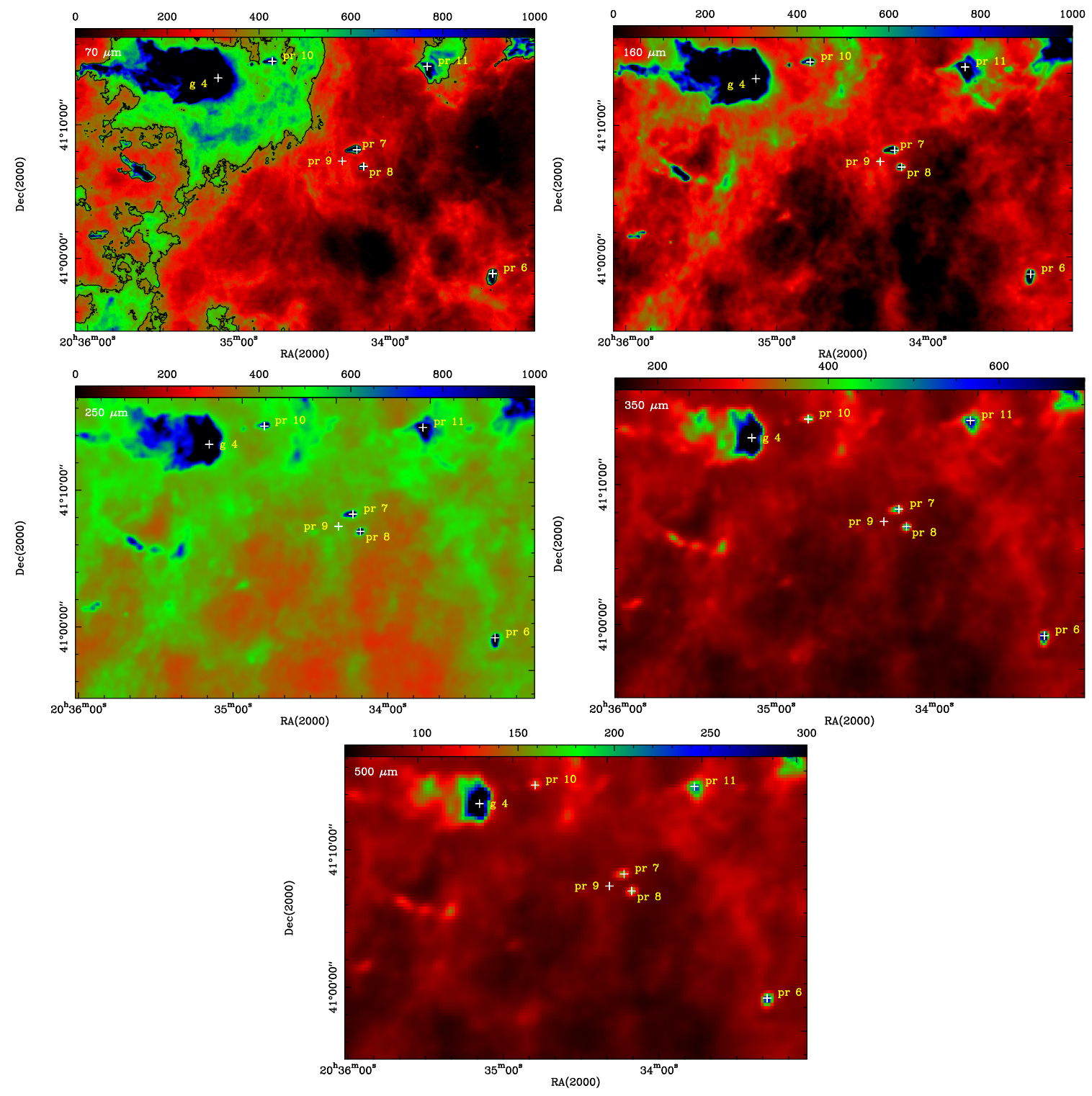

Fig. A.5. PACS (70 and $160 \mu \mathrm{m})$ and $\operatorname{SPIRE}(250,350,500 \mu \mathrm{m})$ images of region 2-2. 
A\&A 591, A40 (2016)
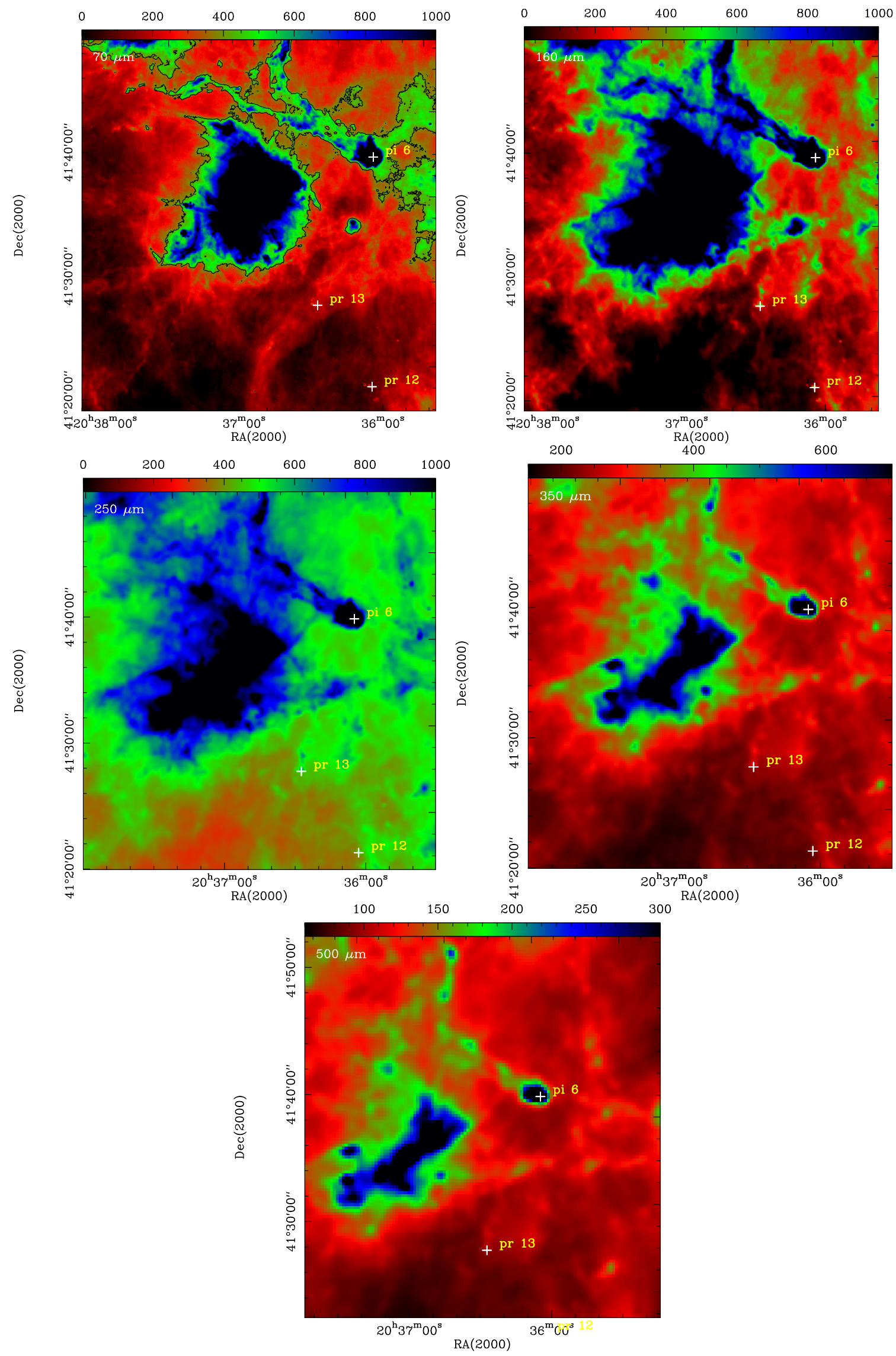

Fig. A.6. PACS (70 and $160 \mu \mathrm{m})$ and $\operatorname{SPIRE}(250,350,500 \mu \mathrm{m})$ images of region 3-1. 
N. Schneider et al.: Far-IR imaging of Cyg OB2

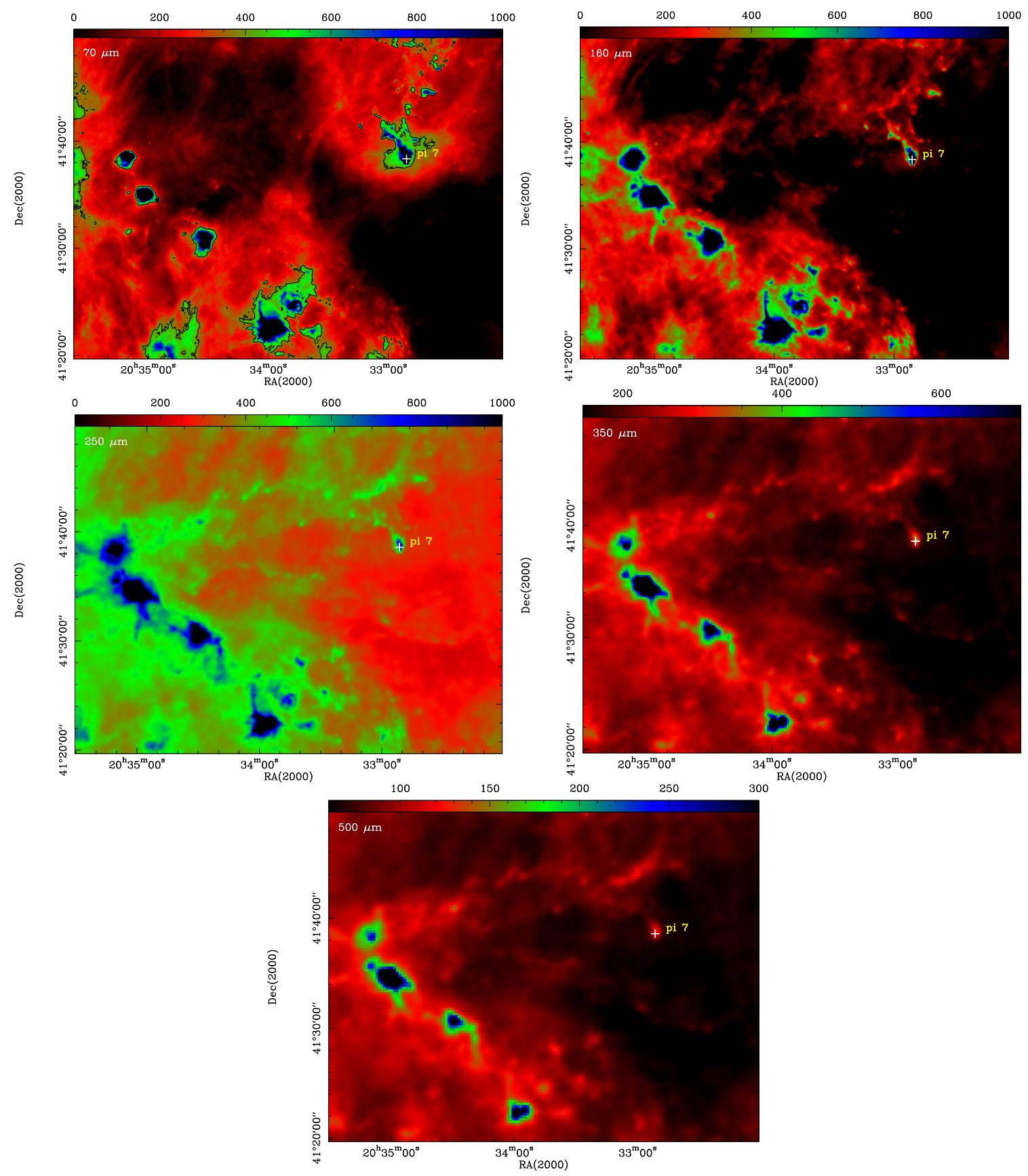

Fig. A.7. PACS (70 and $160 \mu \mathrm{m})$ and $\operatorname{SPIRE}(250,350,500 \mu \mathrm{m})$ images of region 3-2. 